\title{
Ciliary transition zone proteins coordinate ciliary protein composition and ectosome shedding
}

\section{Liang Wang}

Jiangsu Normal University https://orcid.org/0000-0001-5950-0593

\section{Xin Wen}

Jiangsu Normal University

\section{Zhengmao Wang}

Tsinghua University

\section{Zaisheng Lin}

Shanghai Jiao Tong University

\section{Chunhong Li}

Jiangsu Normal University

Huilin Zhou

Jiangsu Normal University

Huimin $\mathrm{Yu}$

Jiangsu Normal University

\section{Yuhan Li}

Jiangsu Normal University

\section{Yifei Cheng}

Jiangsu Normal University

\section{Geer Lou}

Shanghai Biotree Biotech Co. Ltd

Junmin Pan

Tsinghua University https://orcid.org/0000-0003-1242-3791

Muqing Cao ( $\nabla$ muqingcao@sjtu.edu.cn )

Shanghai Jiao Tong University https://orcid.org/0000-0002-0352-6548

\section{Article}

Keywords: cilia, transition zone, TCTN1, ectosome

Posted Date: December 8th, 2021

DOI: https://doi.org/10.21203/rs.3.rs-1142196/v1 
License: (c) (i) This work is licensed under a Creative Commons Attribution 4.0 International License. Read Full License

Version of Record: A version of this preprint was published at Nature Communications on July 9th, 2022. See the published version at https://doi.org/10.1038/s41467-022-31751-0. 
2 Ciliary transition zone proteins coordinate ciliary protein composition and 3 ectosome shedding

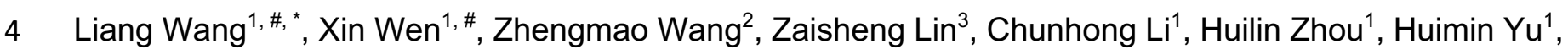

5 Yuhan $\mathrm{Li}^{1}$, Yifei Cheng ${ }^{1}$, Geer Lou ${ }^{4}$, Junmin $\mathrm{Pan}^{2}$, Muqing $\mathrm{Cao}^{3,}{ }^{*}$

$6 \quad{ }^{1}$ School of Life Sciences, Jiangsu Normal University, Xuzhou 221116, China

$7 \quad$ 2School of Life Sciences, Tsinghua University, Beijing 100084, China; Laboratory for Marine Biology and 8 Biotechnology, Qingdao National Laboratory for Marine Science and Technology, Qingdao 266071, 9 China

$10{ }^{3}$ Key Laboratory of Cell Differentiation and Apoptosis of Chinese Ministry of Education, Department of 11 Pathophysiology, Shanghai Jiao Tong University School of Medicine, Shanghai 200025, China

$12{ }^{4}$ Shanghai Biotree Biotech Co. Ltd, Shanghai 201815, China

${ }^{\#}$ L.W., and X.W. contributed equally to this work.

*To whom correspondence may be addressed. Email: wangliang@jsnu.edu.cn, muqingcao@sjtu.edu.cn.

Lead contact: L.W. Email: wangliang@jsnu.edu.cn.

Keywords: cilia; transition zone; TCTN1; ectosome

This PDF file includes: Manuscript Text

Figures 1 to 7 Supplementary Figures 1 to 6 
The transition zone (TZ) of the cilium/flagellum serves as a diffusion barrier that controls the entry/exit of ciliary proteins. Mutations of the TZ proteins disrupt barrier function and lead to multiple human diseases. However, the systematic regulation of ciliary composition and signaling-related processes by different TZ proteins is not completely understood. Here, we reveal that loss of TCTN1 in Chlamydomonas reinhardtii disrupts the assembly of Y-links in the TZ. Proteomic analysis of cilia from WT and three TZ mutants, tctn1, cep290, and nphp4, showed a unique role of each TZ subunit in the regulation of ciliary composition, explaining the phenotypic diversity of different TZ mutants. Interestingly, we found that defects in the TZ impair the formation and biological activity of ciliary ectosomes. Collectively, our findings provide systematic insights into the regulation of ciliary composition by TZ proteins and reveal a link between the TZ and ciliary ectosomes.

\section{Main Text}

\section{Introduction}

Cilia and flagella, conserved antenna-like structures protruding from cell surfaces, harbor a number of signalingassociated molecules that maintain cellular homeostasis and regulate tissue development 1, 2, 3. To control the specific ciliary composition, a specialized zone at the base of cilia named the transition zone (TZ) serves as a gatekeeper to sort proteins into/out of the cilia ${ }^{4,5,6}$. Defects in TZ genes cause a variety of syndromes, termed ciliopathies, including Meckel-Gruber syndrome (MKS), nephronophthisis (NPHP), Joubert syndrome (JBTS), Senior-Loken syndrome (SLSN), and oral-facial-digital (OFD) syndrome, characterized by polydactyly, kidney cysts, ataxia, hyperpnea, nervous system degeneration, and developmental delay 7, 8, 9, 10,11, 12. The function of the TZ structure relies on the integrity of two major complexes located at the TZ region, termed the MKS complex and NPHP complex, including MKS1, TMEM67, TMEM216, B9D1, B9D2, CEP290, and TCTN1-3 for the MKS complex and NPHP1, NPHP4, NPHP8, and NPHP2 for the NPHP complex ${ }^{9,13}$. Defects in any gene encoding a protein in the two complexes have been associated with various human diseases, indicating the indispensable function and different role of each member. To date, how each TZ protein uniquely and systematically controls the sorting of ciliary proteins for ciliary assembly and signaling remains largely unknown.

Ectosomes, vesicles secreted from the cell surface, are composed of various types of proteins and RNAs and mediate cellular communication in cell populations ${ }^{14,15,16,17,18}$. Surprisingly, the ciliary membrane is capable of generating ectosomes, and the shedding of ciliary ectosomes plays multiple roles in biological processes ${ }^{19,} 20,21,22$, 23, 24, 25. Unlike mammalian cells shedding off both ectosomes and exosomes from plasma membrane with distinct assembly mechanisms and diameters, cilia are the unique source of ectosomes derived from $C$. reinhardtii cells surrounded by cell walls, making $C$. reinhardtii as an ideal model for the study on the mechanism of ectosomes shedding $17,19,20$. However, whether the TZ, a major regulator of ciliary components and signaling, has functions in the release of ciliary ectosomes is a potentially interesting and important area of investigation.

In this study, we identified a mutant cell line of $C$. reinhardtii. The disrupted gene encodes a homolog of mammalian TCTN proteins; thus, we named the mutant $t c t n 1$. Our results showed that $C$. reinhardtii TCTN1 localized to the TZ 
region independent of CEP290 and NPHP4, and the loss of TCTN1 largely attenuated the formation of Y-links in the TZ. Taking advantage of cilia isolation in C. reinhardtii, we purified cilia from WT and three TZ mutants, tctn1, nphp4, and cep290. Proteomic analysis of the cilia indicated that each gate molecule played a unique role in the control of ciliary proteins into/out of cilia, although some common functions were observed. Furthermore, we found that disruption of the TZ altered the size and biological activities of ciliary ectosomes. Systematic proteomics profiling and ectosome analysis showed that each TZ protein has a unique function in ciliary events, including ciliary protein sorting and ciliary ectosome shedding.

\section{Results}

\section{Loss of TCTN1 attenuates ciliogenesis in C. reinhardtii}

To identify new genes involved in ciliary assembly, we performed an unbiased forward genetic screen in a mutant library generated by insertional mutagenesis and found one cilia-deficient cell line. Restriction enzyme site-directed PCR (RESDA-PCR) showed that the insertion was located in the fifth exon of the Cre03.g181450 gene (Fig. 1a), which encodes a 679 aa homologous protein of mammalian TCTN1, 2, and 3 (Fig. 1b). Since there is only one TCTN homolog in C. reinhardtii, we named the mutant tctn1. In contrast to wild-type cells with two equal-length cilia, tctn1 cells were palmelloid without visible cilia (Fig. 1c, d), indicating fail of lysis of mother cell wall after mitosis. Transformation of the tctn1 mutant with an HA-tagged full-length TCTN1 DNA fragment restored TCTN1 expression (Supplementary Fig. 1a) and rescued the defect in ciliary assembly (Fig. 1c and Supplementary Fig. 1b), which confirmed that the ciliary phenotype was caused by the mutation in TCTN1. Autolysin, the proteolytic enzyme, is often used for lysis of cell wall to hatch daughter cells ${ }^{19,26}$. Upon autolysin enzyme treatment, tctn 1 cells hatched from the mother cell wall and assembled cilia (Fig. 1e). However, the average final ciliary length after hatching reached $\sim 8 \mu \mathrm{m}$, which was shorter than that of WT cells (Fig. 1f, g), and occasionally bulges at the tip of short cilia were observed (Fig. 1e). It was reported that the TZ functioned in deciliation, ciliary assembly, and disassembly in other organisms ${ }^{27,28,29}$, so we determined the excision, regeneration and resorption of the cilia in the tctn 1 cells. Consistent with the WT and rescued cells, tctn1 cells showed no defects in the deciliation process and completed the ciliary regeneration to reach the original length 2 hours after deciliation by $\mathrm{pH}$ shock, but the mutants showed slower ciliary assembly kinetics (Fig. 1g). Interestingly, in the cilia shortening process induced by Nappi, tctn1 cells showed faster ciliary disassembly kinetics (Fig. 1h).

TCTN1, 2, and 3 localize at the TZ in other model organisms ${ }^{30,31}$. Immunostaining analysis of the rescued cell line expressing TCTN1-HA showed that TCTN1 in C. reinhardtii is also a TZ protein at the base of the cilium (Fig. 1i). Consistently, polyclonal antibodies against endogenous $C$. reinhardtii TCTN1 showed the same TZ staining pattern in the WT and rescued lines but not in the tctn1 mutant (Supplementary Fig. 1c). The polyclonal antibodies against endogenous TCTN1 showed additional signal in the cell body compared with the HA antibody (Fig. 1i and Supplementary Fig. 1c). Because the antibodies against the endogenous TCTN1 showed signals in cell bodies of the tctn1 cells, which lost TCTN1 protein, it was very possible that the staining of the cell body was nonspecific. Consistent with previous studies for other TZ protein, TCTN1 was also associated with the ciliary base after deciliation and reciliation (Supplementary Fig. 1d) ${ }^{32,33}$. Immunoblot analysis of whole cells, cell bodies, and cilia verified that TCTN1 was not present in cilia (Fig. 1j), which is consistent with proteomic studies of cilia and the TZ 
in C. reinhardtii ${ }^{34,35}$. Taken together, these data demonstrated that $C$. reinhardtii TCTN1 is a TZ protein and 102 functions in ciliogenesis.

103

104

105

106

107

108

109

110

111

112

113

114

115

116

117

118

119

120

121

122

123

124

125

126

127

128

129

130

131

132

133

134

135

136

137

138

139

\section{Loss of TCTN1 causes ultrastructural defects in the TZ}

C. reinhardtii is an ideal model for transmission electron microscopy (TEM) studies of the ultrastructure of cilia, especially the TZ. TEM revealed that the bulges in the short cilia were filled with electron-dense material (Fig. 2a). The elongated cilia of tctn1 mutant cells showed a normal ultrastructure with 9+2 microtubule doublets (Supplementary Fig. 2a). In the longitudinal sections through the TZ, Y-links (also called wedge-shaped structures or Y-linkers) were present in WT and rescued cells but were missing in tctn1 cells (Fig. 2b). In the cross sections, the Y-links bridging the TZ microtubules and ciliary membrane were often absent in tctn1 cells (Fig. 2c). Disruption of $Y$-links impairs the connection between the microtubule axoneme and the ciliary membrane ${ }^{33}$. We measured the distance between the $\mathrm{H}$ structure and the ciliary membrane in WT, tctn1 and rescued cells and found that the distance was greater in tctn1 mutant cells than in WT or rescued cells (Fig. 2d).

\section{Localization of TCTN1 in the TZ is independent of CEP290 or NPHP4}

To further illustrate the localization of TCTN1, we performed immunostaining with antibodies against HA, acetylated $\alpha$-tubulin, and CEP290 or NPHP4 and found that TCTN1 was distal to CEP290 but proximal to NPHP4 (Fig. 2e, f). Furthermore, we carried out immunostaining assays in tctn1, cep290, nphp4, and WT cells and found that the localization of TCTN1, CEP290, and NPHP4 was independent of the other two proteins (Fig. $2 \mathrm{~g}$ ).

\section{The ciliary phenotypes of tctn1, cep290, and nphp4 are variable}

The integrity of the TZ affects ciliary morphology and motility. cep290 are often palmelloid and immotile, while nphp4 have normal cilia with slightly defective swimming linearity ${ }^{33,36}$. We compared the phenotypes of tctn 1 , cep290, and nphp4. The tctn1 cells were palmelloid and immotile in TAP medium, similar to cep290 (Supplementary Fig. 2b). After autolysin treatment, tctn1 cells were released from the mother cell wall, and the cilia were gradually assembled. Approximately $90 \%$ of $\operatorname{tcth} 1$ cells had cilia 3 hours after autolysin treatment, and the final average ciliary length was $7.94 \pm 1.14 \mu \mathrm{m}$ (Supplementary Fig. 2b, c). For cep290, $\sim 54 \%$ of cells had cilia with a final average length of $9.47 \pm 0.54 \mu \mathrm{m}$ (Supplementary Fig. $2 \mathrm{~b}, \mathrm{c}$ ). Occasionally, we found that when the mutant tctn1 cells were cultured in M medium (minimal media) at low cell density, the tctn1 cells were not palmelloid, and $\sim 66 \%$ of cells had cilia $(8.81 \pm 0.75 \mu \mathrm{m})$ and swam normally, while this phenotype was not found in cep290 (Supplementary Fig. 2b). Compared to WT cells (ciliary length, $11.42 \pm 0.94 \mu \mathrm{m}$ ) and nphp4 cells (ciliary length, $11.60 \pm 0.98 \mu \mathrm{m}$ ), tctn1 and cep290 cells were paralyzed. Consistently, more ciliary bulges were observed in tctn1 and cep290 cells (Supplementary Fig. 2d). In addition, tctn1 and cep290 cells showed slower ciliary regeneration kinetics than WT and nphp4 cells (Supplementary Fig. 2e). Therefore, these collective data suggest that TCTN1 and CEP290 might play more essential roles in organization of the TZ and sorting of ciliary proteins than NPHP4.

\section{The DUF1619 domain is essential for the function of TCTN1}

DUF1619 (PF07773, IPR011677) is the only conserved domain identified in the TCTN protein family, but the function of this domain is not clear. To determine the function of DUF1619 for TCTN1, we constructed the truncated 


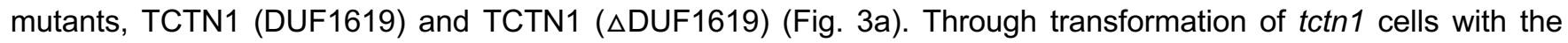
indicated HA-tagged mutants, we generated cell lines with deletion variants (Fig. 3a, b). Transformants were screened for the expression of truncated versions of TCTN1 by immunoblotting (Fig, 3b). The transformants with correct expression were used for the following experiments. Phenotypic analysis showed that two of the deletion mutant transformants examined were palmelloid, which phenocopied the tctn1 mutant (Fig. 3c). Additionally, the ciliary bulges, motility, ciliary length, and ciliary assembly kinetics of the two transformants were comparable to those of tctn 1 cells (Fig. 3d and Supplementary Fig. 3a-c). These data demonstrate that the DUF domain is critical for TCTN1 function.

\section{Loss of TCTN1 systematically disrupts ciliary components}

It has been reported that TCTN proteins contribute to the concentration of several ciliary membrane proteins in mammalian cells and worms ${ }^{31,37,38}$. However, the systematic changes in the ciliary protein composition in TZ mutants are unknown. The cilia of WT and tctn1 were isolated and subjected to silver gel staining and proteomic analysis. Total ciliary protein (Cilia) and the membrane-matrix ( $M+M)$ and axonemal (Axo) fractions from the WT, tctn1 and/or rescued cells were analyzed via SDS-PAGE. Significant differences between the WT and tctn1 cilia were identified (Fig. 4a). To profile the systematic changes in ciliary proteins, we performed proteomic analysis of WT and tctn1 cilia. Unexpectedly, a volcano plot showed that more proteins in cilia were increased in tctn1 (Fig. $4 b)$, which indicates that defects in the TZ not only impaired ciliary membrane protein concentration but also led to loss of the filter function to exclude other proteins (Fig. 4c). Clearly, the histogram showed an increase in IFT complexes, IFT motors, Golgi, chloroplast and mitochondrion proteins in cilia (Fig. 4c). Immunofluorescence and immunoblot analyses confirmed our proteomic data (Fig. 4d, e and Supplementary Fig. 4a, b). Surprisingly, the organelle proteins that presumably are not normally present in cilia but reside in the Golgi, chloroplast, and mitochondrion were transported into the cilia of the tctn1 mutant (Fig. 4c). We verified these data via immunofluorescence and immunoblotting assays with antibodies against PSBC and PSAD (Fig. 4f, g). Immunofluorescence with anti-PSBC and anti-PSAD antibodies showed cup-shaped chloroplasts in cell bodies (Supplementary Fig. 5a, b) and abnormal accumulation of PSBC and PSAD in the cilia of tctn1 cells (Fig. 4g). Collectively, the above results indicate that the defective TZ permitted entry of cytoplasmic proteins into cilia, which was consistent with the presumed function of the TZ in excluding nonciliary proteins and the gating roles of ciliary proteins in cilia.

\section{Different TZ proteins regulate the specific protein composition of cilia}

The phenotypic differences of the TZ mutants suggest that each TZ protein plays specific roles in control of ciliary composition. To systematically assess the functions of the TZ proteins in ciliary composition regulation, we performed proteomic analysis of cilia purified from WT, tctn1, cep290, and nphp4 cells. In total, 2845 proteins were identified, and their relative abundances were determined. A heatmap indicated that the protein profile of each mutant cell was significantly unique (Supplementary Fig. 6a). Although the heatmap pattern of $n p h p 4$ ciliary proteins was similar to that of the WT cilia, $\sim 30.9 \%$ of the proteins showed threefold changes $(>3$ or $<1 / 3$ ) compared to the WT cilia (Supplementary Fig. 6a). Mutations in TCTN1 or CEP290 seemed to cause more severe defects in the TZ, which led to $51.8 \%$ or $60.9 \%$ of proteins showing threefold changes, respectively (Supplementary Fig. 6a). 
Furthermore, according to the protein function, we performed grouped comparisons with WT and TZ mutants (Fig. 5a-d). Cargo transport-related proteins, including IFT/motor/BBS proteins, accumulated in the cilia of tctn1 and cep290 (Fig. 5a), while ciliary structural proteins, such as axonemal components, were downregulated in the cilia of tctn1 and cep290 (Fig. 5b). The regulation of ciliary membrane proteins seemed to be completely disrupted in the cilia of TZ mutants (Fig. 5c). Consistent with the proteomic results for tctn1, the nonciliary proteins from the Golgi, chloroplast, and mitochondrion were mistransported into the cilia of cep290 mutants (Fig. 5d). The amount of ciliary membrane proteins, IFT proteins, and axonemal proteins was confirmed by immunoblot analysis (Supplementary Fig. 6b). Through immunofluorescence, we also verified that the chloroplast proteins of PSBC and PSAD were mislocated in the cilia of cep290 and tctn1 cells (Fig. 5e-g). These results demonstrate that loss of each TZ protein leads to unique and systematic changes in ciliary composition, which account for specific disruptions in ciliary signaling.

\section{TZ proteins regulate the formation of ciliary ectosome during gamete mating}

Cilia-derived ectosomes contain biologically active molecules and have multiple functions in the regulation of ciliary signaling $19,21,23,24,39,40$. From the ciliary proteomics data, we noticed that the amount of ectosome-related proteins was disrupted in the cilia of mutant cells (e.g., FOX1, AGG3, FEA2, AGG4, FAP212, CYN20-1) (Fig. 6a) ${ }^{20}$. formation may be affected by defects in the TZ. Additionally, defects of the TZ mutant in hatching from the mother cell wall also suggested alteration of ectosome formation in the TZ mutant cilia.

In the mating process, gametic cells release large amounts of ectosomes that activate other gametes by binding to the cilia of cells with opposite mating types ${ }^{22,25}$. We mixed gametic cells $(21 \mathrm{gr} \times 6145 \mathrm{c}$ [WT]; tctn $1 \times 6145 \mathrm{c}$ [tctn1]; cep290 × 6145c [cep290]; nphp4 $\times 6145 c$ [nphp4]) at a ratio of 5:1, in which ectosomes from plus gametes constituted the majority. We purified ciliary ectosomes, followed by the negative staining TEM to visualize the morphology of ciliary ectosomes. Images revealed that the diameters of the ectosomes in the TZ mutant groups were significantly smaller than those in the control group (Fig. 6b, c). The average diameter of ectosomes from WT was $198.20 \pm 4.66 \mathrm{~nm}(\mathrm{n}=500)$, while the diameters of the ectosomes from tctn1, cep290, and nphp4 were $87.35 \pm$ $1.98 \mathrm{~nm}(\mathrm{n}=500), 83.99 \pm 1.72 \mathrm{~nm}(\mathrm{n}=500)$, and $134.40 \pm 3.93 \mathrm{~nm}(\mathrm{n}=500)$, respectively (Fig. 6c). Moreover, the size distribution varied greatly among these ectosomes. For tctn1 and cep290, the diameter of most ectosomes was 50-100 nm, while the diameter of most ectosomes of nphp4 was slightly larger, with a distribution range of 50$100 \mathrm{~nm}$ and $100-150 \mathrm{~nm}$ (Fig. 6d). These data indicate that the TZ structure plays a role in the formation of ciliary ectosomes.

\section{Ciliary ectosomes from the TZ mutants showed different biological activity and protein composition}

Our previous studies showed that ciliary ectosomes exhibit biological activity to induce gamete agglutination of single mating type gametes and activate cilium-generated signaling during the mating process ${ }^{22}$. This behavior of single mating type gametes agglutination induced by the ciliary ectosomes is very similar to the bona fide mating agglutination, thus we name this behavior as pseudomating. Hence, the pseudomating is a possible assay to evaluate the bioactivity of the ciliary ectosomes. Thus, another interesting question is whether the activities of the ectosomes from the TZ mutants were altered. To avoid possible contamination with cell debris that may be pelleted 
during ultracentrifugation, we performed ectosome purification using a commercial exosome/ectosome isolation kit, which specifically precipitated the membrane vesicles upon relatively low-speed centrifugation (at 10,000 $\times g$ ). As denoted in Material and Methods, we conducted a gamete pseudomating assay with the fractions of S20, Sup and Eco, and found that the purified ectosomes, but not the supernatant fraction, possessed the ability to induce the adherence of $6145 \mathrm{c}$ gametic cells, not $6145 \mathrm{c}$ vegetative cells or $6145 \mathrm{c}$ gametic cells alone (Fig. $7 \mathrm{a}, \mathrm{b}$ ). After mixing with the ectosomes for 10 minutes, we measured the percentages of the adhering minus gametes (6145c) and found that the efficiency of the ectosomes from the TZ mutants, especially from the tctn1 or cep290 group, decreased dramatically (Fig. 7c). Consistent with Fig. 7B, the ectosomes failed to induce agglutination of vegetative $6145 \mathrm{c}$ cells in Con group (Fig. 7c). As expected, SDS-PAGE with silver staining showed variations in the protein composition and amount of ectosomes from the four groups (Fig. 7d). Thus, these data further demonstrate that the TZ also plays roles in regulation of the biological activity and protein composition of ciliary ectosomes.

\section{Discussion}

As the only transport route from the cell body to the cilium, the TZ compartment plays essential roles in the regulation of ciliary composition, which fundamentally defines the biological processes and functions of the cilium. Although the structure and composition of the TZ have been characterized, the unique roles of TZ proteins in assembly of the TZ and their systematic regulation of ciliary components are largely unknown. Consistent with previous studies in mammals and worms 9, 30,35,41,42,43,44,45,46, we found that $C$. reinhardtii TCTN1 localizes in the TZ (Fig. 1i, j). Furthermore, we provide both ultrastructural insights and systematic proteomic information upon the loss of TCTN1. Using immunofluorescence microscopy, we showed that TCTN1 localized in the middle region of the TZ independent of CEP290 and NPHP4 (Fig. 2e, f). Using TEM, we found that TCTN1 is essential for assembly of the Y-links in the TZ, similar to CEP290 (Fig. 2b-d) ${ }^{33}$. Previous studies indicated that TCTN1 is located at the ciliary membrane and CEP290 is located near the axoneme ${ }^{47}$. Based on our data, we speculate that TCTN1 and CEP290 may function as anchors of the Y-links at the two ends, and loss of either of the two key components thoroughly disrupts the structure.

The TZ strictly regulates the entry and retention of ciliary proteins and exclusion of nonciliary proteins 9, 33, 43, 48, 49 . It is known that the severity of TZ defects caused by mutations in individual TZ proteins varies, reflecting the different functions of individual proteins in organizing the TZ. Consistently, our phenotypic analysis of the three TZ mutants (tctn1, cep290, and nphp4) also showed various ciliary phenotypes (Supplementary Fig. 2b-e), which were caused by the different systematic changes in the ciliary components (Supplementary Fig. 6a). To date, how the loss of a single TZ protein systematically affects ciliary composition is not clear. Taking advantage of cilia purification from Chlamydomonas, we isolated the cilia of WT, tctn1, cep290, and nphp4 cells and subjected the ciliary samples to proteomic analysis. In agreement with the variable phenotypes, the proteomic profile of cilia from each cell type was quite unique (Fig. 5a-d and Supplementary Fig. 6a). The concentrations of ciliary membrane-associated proteins, including PKD, FOX1, AGG4, and CYN20-1, were disrupted in tctn1, cep290, and nphp4 mutant cilia. Considering the function of membrane proteins, ciliary signaling should be misregulated. Although mutants displayed characteristic protein profiles and the protein ratios were variable in different mutants, we indeed observed several common features shared by the three TZ mutants. First, the proteomic data showed accumulation of IFT subunits and several IFT motors and reduction of the axonemal components in the mutant cilia. Second, nonciliary proteins, 
including various ATP synthases (ATP1A, ATP2, ATPC, and ASA4), photosystem proteins (PSAD, PABC, PSAA, and $P S A B$ ), vesicular transport proteins (Coatomer subunits), and translation initiation factors (EIF3 subunits), also accumulated to different degrees, which is direct evidence of the function of the TZ to exclude nonciliary components. However, thus far, we do not know how these nonciliary proteins could pass through the ciliary gate and whether they have functions in cilia. Furthermore, according to the above phenotypic analysis and ciliary protein profiling in these three TZ mutants (tctn1, cep290, nphp4), we conclude that the different roles of TCTN1, CEP290 and NPHP4 in protein sorting ultimately influence the phenotype. The hierarchy of degrees of phenotypic severity in the three TZ genes is CEP290 > TCTN1 > NPHP4. How they coordinately influence ciliary composition and ciliary functions remains to be further investigated.

Another interesting phenomenon observed in our data inspire us that there may be a link between the TZ proteins and ciliary ectosomes. First, the cellular morphology of TZ mutants (tctn1 and cep290) are palmelloid (Fig. 1c and Supplementary Fig. 2b) ${ }^{33}$, indicating the deficient of ciliary ectosome associated lysis protease shedding within the mother cell walls after cell division ${ }^{19}$. Second, the proteomic analysis was changes in ciliary ectosome-associated proteins (e.g., FOX1, AGG4, CYN20-1) (Fig. 6a) ${ }^{20}$. Until now, it has been unknown whether the TZ structure controls the formation of ciliary ectosomes. Here, we provide direct evidence that defines the function of the TZ in ectosome biogenesis. Loss of the integrity of the TZ affects the morphology, protein composition, and biological activities of ciliary ectosomes (Fig. 6 and Fig. 7). In the future, it will be worth investigating the mechanisms through which TZ proteins influence the amount of ectosome-associated proteins in cilia and the control of ectosome formation and biological activity.

In summary, our work showed that TCTN1 is essential for proper assembly of the Y-links of the TZ. Using proteomic analysis of cilia from WT, tctn1, cep290, and $n p h p 4$ cells, we systematically characterized the ciliary protein profile of the mutants, which revealed the unique function of each TZ protein. Most importantly, a novel function of the TZ in ciliary ectosome formation was uncovered.

\section{Methods}

\section{Strains and cell culture}

C. reinhardtii wild-type strains $21 \mathrm{gr}$ (CC-1690, wild-type, $\mathrm{mt}+$ ) and $6145 \mathrm{C}$ (CC-2895, wild-type, mt-) and the mutant strains cep290 (CC-4374, mt+) and nphp4 (CC-5113, mt+) were provided by the Chlamydomonas Resource Center (University of Minnesota, USA). The tctn1 mutant was generated from the wild-type strain $21 \mathrm{gr}$ by insertional mutagenesis with the paromomycin-resistant DNA fragment aphVIII. Strains were cultured in Tris-acetatephosphate (TAP) plates or liquid medium with aeration at $23 \pm 0.5^{\circ} \mathrm{C}$ with a light/dark cycle of $14 / 10 \mathrm{~h}$ at a light intensity of 8000 Ix. For transformation experiments, the indicated cell line was grown in TAP liquid medium under continuous light. Autolysin was used to release tctn1 and cep290 mutant cells from the mother cell wall and to induce ciliogenesis. For the induction of gametogenesis, vegetatively growing cells were transferred to $\mathrm{N}$-free medium as previously described ${ }^{22}$. For tctn1 or cep290 gametes, autolysin was applied to induce the release of gametes from the palmelloid before mating. The cells were thoroughly washed to remove autolysin and allowed to assemble cilia for $\sim 2 \mathrm{~h}$.

\section{DNA manipulations, transformation, and mutagenesis}


A library of mutants was generated via random insertional using electroporation with the aphVIII gene (paromomycin-resistant cassette, $\sim 2.1 \mathrm{~kb}$ fragment cut with EcoRI from the plasmid pJMG-aphVIII) ${ }^{50}$. Electroporation was performed as described previously ${ }^{50}$. Briefly, cells for transformation were cultured in liquid TAP medium with constant aeration and continuous light until the cell concentration reached $\sim 1.0 \times 10^{7}$ cells $/ \mathrm{mL}$. Then, the cells were inoculated into fresh liquid TAP medium and grown under continuous light for 18-20 $\mathrm{h}$ until the cell concentration reached $\sim 4.0 \times 10^{6} \mathrm{cells} / \mathrm{mL}$. Cells were transformed with the DNA fragment via square-wave electroporation with a BTX ECM830 electroporation apparatus (500 V, 4 ms, 6 pulses) (BTX, USA). The flanking sequences of the aphVIII insertional site were determined by restriction enzyme site-directed PCR (RESDA-PCR) 51, sequencing and blasting with Chlamydomonas reinhardtii v5.5 genome database (https://phytozome.jgi.doe.gov/pz/portal.html\#). For the gene complementation experiments, full-length DNA harboring the TCTN1 locus (with an endogenous promoter) was inserted into the expression construct pHyg-3HA. For construction of deletion mutants of the TCTN1 gene, all deletion mutant constructs were generated based on the wild-type TCTN1 construct and are indicated in the text. The resulting constructs were then linearized with Ndel (Takara, Japan) and transformed into tctn1 mutant with electroporation. The transformants were plated onto the hygromycin (Sigma, USA) plates and screened for the ciliary phenotypes and via immunoblot analysis.

\section{Analysis of ciliary and cellular phenotypes}

The liquid-medium-cultured cells were put on the slide, and observed the cell status with inverted microscope. To capture the cell images, cells were fixed with $5 \%$ glutaraldehyde solution, plated on the slide with a cover-slip, observed and captured by LAS V4.0 imaging software on a Leica DMI4000 B Inverted Microscope (Leica, Germany) with a $10 \times, 20 \times, 40 \times$ or $63 \times$ objective (HCX PL FLUOTAR $10 \times / 0.30,506507$; HCX PL FLUOTAR L $20 \times / 0.40$, 506243; HCX PL FLUOTAR L 40 × / 0.60, 506203; HCX PL FLUOTAR L $63 \times / 0.70,506217$ ) for differential interference contrast (DIC)/ phase contrast $(\mathrm{PH})$ images. The ciliary length was measured for 50 cells by Image J (National Institutes of Health, USA). The cell number was counted for 200 cells. All original data were analyzed and plotted with GraphPad Prism (GraphPad Software, USA). All experiments were repeated three times.

\section{Ciliary deciliation, regeneration, and resorption}

To induce the ciliary deciliation, cells was deciliated by $\mathrm{pH}$ shock method ${ }^{52,53}$. Briefly, cells were quickly treated with $0.5 \mathrm{M}$ glacial acetic acid, kept at $\mathrm{pH} \sim 4.5$ for $30 \mathrm{~s}$, then quickly added by $0.5 \mathrm{M} \mathrm{KOH}$ to recover the $\mathrm{pH} \sim 7.0$. To induce regeneration of the cilia, cells after deciliation were centrifuged and washed with fresh $\mathrm{M}$ medium to be allowed for ciliary regeneration with aeration at $23 \pm 0.5^{\circ} \mathrm{C}$ in light. To induce resorption of the cilia, cells incubated

with $20 \mathrm{mM}$ sodium pyrophosphate (NaPPi) in M medium gradually shorten cilia over time ${ }^{54}$. At the indicated time point, cells were collected for imaging and measurement of ciliary length or for the immunoblot analysis. All experiments were repeated three times.

\section{Autolysin preparation}

The cell wall proteolytic enzyme autolysin is typically generated during plus and minus gametes mating ${ }^{26}$. Generally, we followed the gamete autolysin preparation procedure (https://www.chlamycollection.org/methods/preparationof-gamete-autolysin/), except M-N medium for nitrogen starvation was used to induce gamete differentiation. Then 
Ix). Equal amount of opposite mating type gametes $(21 \mathrm{gr} \times 6145 \mathrm{c})$ were mixed to allow mating with gentle aeration for $\sim 15 \mathrm{~min}$. After centrifugation at $600 \times \mathrm{g}$, for $5 \mathrm{~min}$ and $14,000 \times \mathrm{g}$, for $10 \mathrm{~min}$ respectively, the final supernatant was filtrated by $0.22 \mu \mathrm{m}$ membrane filter and aliquot, stored at $-80^{\circ} \mathrm{C}$ until use.

\section{Isolation and fractionation of cilia and ciliary ectosomes}

To isolate cilia, cells were deciliated by $\mathrm{pH}$ shock. Detached cilia were enriched via sucrose gradient centrifugation and pelleted by high-speed centrifugation ${ }^{55}$. To fractionate the ciliary samples into the $M+M$ (membrane and matrix) fraction and Axo (axoneme) fraction, the ciliary pellet was lysed in 0.5\% NP40 buffer A (50 mM Tris- $\mathrm{HCl}$ [pH 7.5], $10 \mathrm{mM} \mathrm{MgCl}$, $1 \mathrm{mM}$ EDTA, $1 \mathrm{mM}$ DTT) with EDTA-free protease inhibitor cocktail (Roche, Switzerland), frozen in liquid nitrogen and thawed, followed by centrifugation at $20,000 \times g$ at $4{ }^{\circ} \mathrm{C}$ for $10 \mathrm{~min}$. The supernatants and pellets were the $\mathrm{M}+\mathrm{M}$ fraction and Axo fraction, respectively.

Isolation of ciliary ectosomes by ultracentrifugation was carried out as described previously ${ }^{22}$ with slight modifications. Briefly, The mating type plus $(\mathrm{mt}+)$ gametes $(21 \mathrm{gr}, \mathrm{tctn} 1$, cep290, and nphp4) are mixed with the mating type minus (mt-) gametes 6145c (mt+: mt- =5: 1) for $15 \mathrm{~min}$, followed by $600 \times \mathrm{g}$ for $5 \mathrm{~min}, 20,000 \times \mathrm{g}$ for $10 \mathrm{~min}$, and the final step of $150,000 \times \mathrm{g}, 60 \mathrm{~min}$ to obtain the ectosome pellet (Eco). The ciliary ectosomes were also purified with a Total Exosome Isolation (from cell culture media) Kit (Cat. No. 4478359, ThermoFisher, USA). The media with above described mating cells were centrifuged at $600 \times g$ for 5 min and 20,000 $\times g$ for 5 min ( 3 times) to remove the cells and debris. The resulting supernatant (S20) was finally incubated in buffer at $4{ }^{\circ} \mathrm{C}$ for 2 $\mathrm{h}$ and then pelleted by centrifugation at $10,000 \times \mathrm{g}$ for $60 \mathrm{~min}$ to obtain the final supernatant (Sup) and ectosome pellet (Eco). The pelleted ectosomes were resuspended in $\mathrm{N}$-free medium. The ectosomes were image by TEM. At least 500 ectosomes were measured for their diameters by ImageJ software for each group. The experiments were performed for three times.

\section{Assay for ectosome activity}

To determine the activity of ectosomes, purified ectosomes were applied for gamete agglutination with 6145c. In particular, ectosomes from different mating events $(21 \mathrm{gr}$, tctn1, cep290, or $n p h p 4 \times 6145 \mathrm{c})$ were mixed with $6145 \mathrm{c}$ gametes $\left(10 \mu \mathrm{L}, 1.0 \times 10^{8} \mathrm{cells} / \mathrm{mL}\right)$ to induce agglutination for $10 \mathrm{~min}$. The agglutinating gametes were observed and imaged alive. The ratios of cell adhesion were plotted using GraphPad Prism software (GraphPad Software, USA).

\section{Immunofluorescence microscopy}

Immunofluorescence was carried out essentially as previously described ${ }^{52}$. For detection of CEP290 and NPHP4, cells were collected and resuspended in MT buffer (30 mM HEPES [pH 7.2], 3 mM EGTA, 1 mM MgSO, 25 mM $\mathrm{KCl}$ ), followed by fixation and extraction in $100 \%$ prechilled methanol for $20 \mathrm{~min}$ at $-20{ }^{\circ} \mathrm{C}$. For detection of other proteins, cells were collected and resuspended in MT buffer for 2 min, followed by fixation in $4 \%$ paraformaldehyde fixation solution (Biosharp, China) for $5 \mathrm{~min}$ at room temperature. After the fixation step, cells were resuspended in MT buffer with $0.5 \% \mathrm{NP}-40$ for 2 min and further extracted in $100 \%$ prechilled methanol for $10-15$ min at $-20{ }^{\circ} \mathrm{C}$. The cells were then sequentially rehydrated with PBS, blocked with goat blocking buffer (containing $5 \%$ goat serum) (Sangon Biotech, China), and incubated with primary antibodies at $4{ }^{\circ} \mathrm{C}$ overnight, followed by incubation with secondary antibodies at $37^{\circ} \mathrm{C}$ for $2 \mathrm{~h}$. 
The secondary antibodies used were preadsorbed anti-rat IgG H\&L (Alexa Fluor ${ }^{\circledR} 488$ ), preadsorbed anti-mouse IgG H\&L (Alexa Fluor® 594), preadsorbed anti-rabbit IgG H\&L (Alexa Fluor® 647), preadsorbed anti-rabbit IgG H\&L (Alexa Fluor® 594), preadsorbed anti-rabbit IgG H\&L (Alexa Fluor ${ }^{8} 488$ ), and preadsorbed anti-mouse IgG H\&L (Alexa Fluor® 647) (Abcam, UK). The secondary antibodies were used at a dilution of 1:500.

After three washes for 5 min with 0.5\% Tween-20 PBS, one wash with PBS, and one wash with Milli-Q, samples on the slides were mounted with DAPI-Fluoromount-G (SouthernBiotech, USA), sealed with nail polish and air dried for $\sim 2 \mathrm{~h}$ before observation. Samples were then imaged on a Leica TCS SP5-II or Leica TCS SP8 confocal laser microscope (Leica, Germany) with a 63 × oil immersion objective (HCX PL APO 63 × / 1.40-0.60 OIL CS, 506188). Images were acquired and processed using Leica Application Suite AF or Leica Application Suite $X$ and Adobe Photoshop and assembled using Adobe Illustrator (Adobe Systems Incorporated, USA).

\section{SDS-PAGE and immunoblotting}

Cells were harvested by centrifugation at $10,000 \times g$ for $1 \mathrm{~min}$, frozen in liquid nitrogen and stored at $-80^{\circ} \mathrm{C}$ until use. The frozen samples were then lysed in prechilled buffer $A(50 \mathrm{mM}$ Tris- $\mathrm{HCl}[\mathrm{pH} 7.5], 10 \mathrm{mM} \mathrm{MgCl}, 1 \mathrm{mM}$ EDTA, 1 mM DTT) with EDTA-free protease inhibitor cocktail (Roche, Switzerland) and boiled in $1 \times$ SDS sample buffer for $5 \mathrm{~min}$ before being subjected to SDS-PAGE and blotting analysis. The secondary antibodies used were HRP-conjugated goat anti-mouse, goat anti-rabbit and goat anti-rat (1:5000; Jackson, USA). The final immunoreactive bands were visualized and analyzed using an Amersham imager 600 (GE, USA).

\section{Primary antibodies}

The primary antibodies used for immunoblotting (IB) or immunofluorescence (IF) were as follows: anti-HA high affinity (3F10, rat monoclonal IgG, 1:3000 for IB and 1:50 for IF; Roche, Switzerland), anti-a-tubulin (mouse monoclonal IgG, 1:5000 for IB and 1:200 for IF; Proteintech, USA), anti-a-tubulin (rabbit monoclonal IgG, 1:5000 for IB; Proteintech, USA), anti-centrin (clone 20H5, mouse monoclonal IgG, 1:400 for IF; Merck Millipore, Germany), anti-acetylated $\alpha$-tubulin (clone 6-11B-1, mouse monoclonal IgG, 1:200 for IF; Sigma, USA), anti-CEP290 (rabbit polyclonal IgG, 1:200 for IF; a gift from Dr. George Witman), anti-NPHP4 (rabbit polyclonal IgG, 1:100 for IF; a gift from Dr. George Witman), anti-FMG-1B (\#61, mouse monoclonal IgG, 1:100 for IB, Developmental Studies Hybridoma Bank), anti-IFT122 (rabbit polyclonal IgG, 1:2000 for IB and 1:100 for IF), anti-IFT121 (rabbit polyclonal serum, 1:2000 for IB), anti-IFT172 (rabbit polyclonal IgG, 1:2000 for IB), anti-IFT57 (rabbit polyclonal IgG, 1:2000 for IB and 1:100 for IF), anti-IFT54 (rabbit polyclonal serum, 1:2000 for IB), anti-IFT43 (rabbit polyclonal serum, 1:100 for IF), anti-IFT38 (rabbit polyclonal IgG, 1:5000 for IB and 1:100 for IF), anti-FLA10 (rabbit polyclonal IgG, 1:3000 for IB), anti-D1BLIC (rabbit polyclonal IgG, 1:2000 for IB), anti-BBS8 (rabbit polyclonal IgG, 1:250 for IB), anti-RIB72 (rabbit polyclonal IgG, 1:3000 for IB), anti-IC2 (mouse monoclonal IgG, 1:20000 for IB; Sigma, USA), anti-PSAD (rabbit polyclonal serum, 1:1000 for IB, 1:500 for IF; a gift from Dr. Xiaobo Li; Agrisera, Sweden), and anti-PSBC (rabbit polyclonal serum, 1:3000 for IB, 1:500 for IF; a gift from Dr. Xiaobo Li; Agrisera, Sweden). A rabbit anti-TCTN1 antibody was generated against bacterial expressed GST-His-tagged TCTN1 (368-490 amino acids) (Abclonal, China) and was only used at a dilution of 1:500 for IF.

\section{Transmission electron microscopy (TEM)}


417 Negative staining and TEM were carried out as previously described ${ }^{22,55}$. The samples were imaged on an $\mathrm{H}$ -

418 7650B transmission electron microscope (Hitachi Limited, Japan) equipped with a digital V600 camera (ATM 419 Company).

420

421

422

423

424

425

426

427

428

429

430

431

432

433

434

435

436

437

438

439

440

441

442

443

444

445

446

447

448

449

450

451

452

453

454

\section{Quantitative proteomics}

A total of $150 \mu \mathrm{g}$ of ciliary proteins from each sample was dissolved in RIPA buffer containing protease inhibitor. The dissolved proteins were precipitated and washed with acetone solution. The concentration of redissolved samples was determined with a bicinchoninic acid protein quantification kit (Fisher Scientific, MA, USA). Then, 100 $\mu \mathrm{g}$ of protein was reduced with $5 \mathrm{mM}$ dithiothreitol and alkylated with $10 \mathrm{mM}$ iodoacetamide in $100 \mathrm{mM}$ HEPES with $1 \%$ SDC. Trypsin $(1 \mu \mathrm{g})$ was used for protein digestion overnight at $37^{\circ} \mathrm{C}$. SDC and salt were removed, and the peptides were dried in a freeze dryer. The peptides, resuspended in $0.1 \%$ TFA, were separated using a ReprosilPur $120 \mathrm{C} 18$ analytical column (100 $\mu \mathrm{m}$ ID $\times 15 \mathrm{~cm}, 1.9 \mu \mathrm{m}$, Dr. Maisch) on a nano-UPLC system (EASY-nLC1200). Then, $0.1 \%$ formic acid in acetonitrile/water (2:98) was used as mobile phase $A$, and $0.1 \%$ formic acid in acetonitrile/water (80:20) was used as mobile phase B. A Q-Exactive HFX mass spectrometer (Thermo Scientific, MA, USA) was operated in data-dependent acquisition mode for sample analysis. MS1 was performed in a range of $350-1600 \mathrm{~m} / \mathrm{z}$ with a resolution of $120,000(200 \mathrm{~m} / \mathrm{z})$. The top 20 precursor ions were fragmented by high-energy C-trap dissociation (HCD) with a normalized collision energy (NCE) of $27 \%$. In MS/MS, the resolution was set to 15,000 , the $A G C$ control was $1.0 \times 10^{5}$, the maximum ion introduction time was $110 \mathrm{~ms}$, and the dynamic exclusion time was 45 seconds. The MS/MS spectra from each run were searched against the species-level UniProt FASTA databases (Chlamydomonas reinhardtii, 2020-01-11, total entries 31247, reviewed entries 339, unreviewed entries 30908). The raw data were processed using Proteome Discoverer (PD) software. The search criteria were as follows: tryptic digestion, 2 missed cleavages allowed, carbamidomethyl (C) set as a fixed modification, and oxidation (M) and acetyl (protein N-term) set as variable modifications. Peptide identification was carried out with an initial precursor ion mass deviation of up to $10 \mathrm{ppm}$ and a fragment mass deviation of $0.02 \mathrm{Da}$. For protein identification, the false discovery rate (FDR) was set at 0.01 for both peptide spectral matches (PSMs) and peptide levels. Total peptide was used for normalization, and both unique peptides and razor peptides were used for further quantification. Other parameters were set as default.

\section{Acknowledgments}

We thank Dr. George Witman and Dr. Xiaobo Li for kindly providing antibodies. We would also like to thank Dr. Jun Lu and Dr. Yuanlin Zheng for equipment support. We are grateful to the Center of Biomedical Analysis (Tsinghua University, China) for their excellent help with the electron microscopy analysis.

This work was supported by the National Natural Science Foundation of China (91954123, 31972887, and 31972888), the Natural Science Foundation of Jiangsu Province (BK20191465), Clinical Research Projects of Shanghai Municipal Health Commission (20194Y0133), and the Priority Academic Program Development of Jiangsu Higher Education Institutions (PAPD).

\section{Author Contributions}


L.W., X.W., and M.C. designed, performed the experiments, and analyzed the data. Z.L., C.L., H.Z., H.Y., Y.L., Y.C., and Z.W. performed the experiments. G.L. analyzed the data. J.P., L.W., and M.C. conceived the idea, analyzed the data and wrote the manuscript. All authors approved the final manuscript.

\section{Competing Interest Statement}

The authors declare no competing interests.

\section{Data availability}

The data that support the findings of this study are available from the corresponding author upon reasonable request.

\section{References}

1. Ishikawa H, Marshall WF. Ciliogenesis: building the cell's antenna. Nat Rev Mol Cell Biol 12, 222-234 (2011).

2. Satir P. CILIA: before and after. Cilia 6, 1 (2017).

3. Bayless BA, Navarro FM, Winey M. Motile Cilia: Innovation and Insight From Ciliate Model Organisms. Front Cell Dev Biol 7, 265 (2019).

4. Lechtreck KF. IFT-Cargo Interactions and Protein Transport in Cilia. Trends Biochem Sci 40, 765-778 (2015).

5. Nachury MV. The molecular machines that traffic signaling receptors into and out of cilia. Curr Opin Cell Biol 51, 124-131 (2018).

6. Takao D, Wang L, Boss A, Verhey KJ. Protein Interaction Analysis Provides a Map of the Spatial and Temporal Organization of the Ciliary Gating Zone. Curr Biol 27, 2296-2306 e2293 (2017).

7. Sreekumar V, Norris DP. Cilia and development. Curr Opin Genet Dev 56, 15-21 (2019).

8. Youn YH, Han YG. Primary Cilia in Brain Development and Diseases. Am J Pathol 188, 11-22 (2018).

9. Garcia-Gonzalo FR, Reiter JF. Open Sesame: How Transition Fibers and the Transition Zone Control Ciliary Composition. Cold Spring Harb Perspect Biol 9, (2017).

10. Mitchison HM, Valente EM. Motile and non-motile cilia in human pathology: from function to phenotypes. $J$ Pathol 241, 294-309 (2017).

11. Anvarian Z, Mykytyn K, Mukhopadhyay S, Pedersen LB, Christensen ST. Cellular signalling by primary cilia in development, organ function and disease. Nat Rev Nephrol 15, 199-219 (2019).

12. Wallmeier J, et al. Motile ciliopathies. Nat Rev Dis Primers 6, 77 (2020).

13. Reiter JF, Leroux MR. Genes and molecular pathways underpinning ciliopathies. Nat Rev Mol Cell Biol 18, 533-547 (2017).

14. Johnstone RM, Adam M, Hammond JR, Orr L, Turbide C. Vesicle formation during reticulocyte maturation. Association of plasma membrane activities with released vesicles (exosomes). J Biol Chem 262, 9412-9420 (1987).

15. Heijnen HF, Schiel AE, Fijnheer R, Geuze HJ, Sixma JJ. Activated platelets release two types of membrane vesicles: microvesicles by surface shedding and exosomes derived from exocytosis of multivesicular bodies and alpha-granules. Blood 94, 3791-3799 (1999).

16. Denzer K, Kleijmeer MJ, Heijnen HF, Stoorvogel W, Geuze HJ. Exosome: from internal vesicle of the multivesicular body to intercellular signaling device. J Cell Sci 113 Pt 19, 3365-3374 (2000). 
17. Cocucci E, Meldolesi J. Ectosomes and exosomes: shedding the confusion between extracellular vesicles. Trends Cell Biol 25, 364-372 (2015).

18. van Niel G, D'Angelo G, Raposo G. Shedding light on the cell biology of extracellular vesicles. Nat Rev Mol Cell Biol 19, 213-228 (2018).

19. Wood CR, Huang K, Diener DR, Rosenbaum JL. The cilium secretes bioactive ectosomes. Curr Biol 23, 906911 (2013).

20. Long H, et al. Comparative Analysis of Ciliary Membranes and Ectosomes. Curr Biol 26, 3327-3335 (2016).

21. Wood CR, Rosenbaum JL. Ciliary ectosomes: transmissions from the cell's antenna. Trends Cell Biol 25, 276285 (2015).

22. Cao M, et al. Uni-directional ciliary membrane protein trafficking by a cytoplasmic retrograde IFT motor and ciliary ectosome shedding. Elife 4, (2015).

23. Nager AR, et al. An Actin Network Dispatches Ciliary GPCRs into Extracellular Vesicles to Modulate Signaling. Cell 168, 252-263 e214 (2017).

24. Phua SC, et al. Dynamic Remodeling of Membrane Composition Drives Cell Cycle through Primary Cilia Excision. Cell 168, 264-279 e215 (2017).

25. Luxmi R, Kumar D, Mains RE, King SM, Eipper BA. Cilia-based peptidergic signaling. PLoS Biol 17, e3000566 (2019).

26. Matsuda Y, Saito T, Yamaguchi T, Koseki M, Hayashi K. Topography of cell wall lytic enzyme in Chlamydomonas reinhardtii: form and location of the stored enzyme in vegetative cell and gamete. J Cell Biol 104, 321-329 (1987).

27. Gogendeau D, et al. MKS-NPHP module proteins control ciliary shedding at the transition zone. PLoS Biol 18, e3000640 (2020).

28. Shimada H, et al. In Vitro Modeling Using Ciliopathy-Patient-Derived Cells Reveals Distinct Cilia Dysfunctions Caused by CEP290 Mutations. Cell Rep 20, 384-396 (2017).

29. Li A, et al. Ciliary transition zone activation of phosphorylated Tctex-1 controls ciliary resorption, S-phase entry and fate of neural progenitors. Nat Cell Biol 13, 402-411 (2011).

30. Garcia-Gonzalo FR, et al. A transition zone complex regulates mammalian ciliogenesis and ciliary membrane composition. Nat Genet 43, 776-784 (2011).

31. Yee LE, et al. Conserved Genetic Interactions between Ciliopathy Complexes Cooperatively Support Ciliogenesis and Ciliary Signaling. PLoS Genet 11, e1005627 (2015).

32. Sanders MA, Salisbury JL. Centrin-mediated microtubule severing during flagellar excision in Chlamydomonas reinhardtii. J Cell Biol 108, 1751-1760 (1989).

33. Craige B, et al. CEP290 tethers flagellar transition zone microtubules to the membrane and regulates flagellar protein content. J Cell Biol 190, 927-940 (2010).

34. Pazour GJ, Agrin N, Leszyk J, Witman GB. Proteomic analysis of a eukaryotic cilium. J Cell Biol 170, 103-113 (2005).

35. Diener DR, Lupetti P, Rosenbaum JL. Proteomic analysis of isolated ciliary transition zones reveals the presence of ESCRT proteins. Curr Biol 25, 379-384 (2015).

36. Awata J, et al. NPHP4 controls ciliary trafficking of membrane proteins and large soluble proteins at the transition zone. J Cell Sci 127, 4714-4727 (2014). 
37. Weng RR, Yang TT, Huang CE, Chang CW, Wang WJ, Liao JC. Super-Resolution Imaging Reveals TCTN2 Depletion-Induced IFT88 Lumen Leakage and Ciliary Weakening. Biophys J 115, 263-275 (2018).

38. Wang C, Li J, Meng Q, Wang B. Three Tctn proteins are functionally conserved in the regulation of neural tube patterning and Gli3 processing but not ciliogenesis and Hedgehog signaling in the mouse. Dev Biol 430, 156-165 (2017).

39. Wang J, et al. Sensory cilia act as a specialized venue for regulated extracellular vesicle biogenesis and signaling. Curr Biol, (2021).

40. Long H, Huang K. Transport of Ciliary Membrane Proteins. Front Cell Dev Biol 7, 381 (2019).

41. Sang L, et al. Mapping the NPHP-JBTS-MKS protein network reveals ciliopathy disease genes and pathways. Cell 145, 513-528 (2011).

42. Li C, et al. MKS5 and CEP290 Dependent Assembly Pathway of the Ciliary Transition Zone. PLoS Biol 14, e1002416 (2016).

43. Williams CL, et al. MKS and NPHP modules cooperate to establish basal body/transition zone membrane associations and ciliary gate function during ciliogenesis. J Cell Biol 192, 1023-1041 (2011).

44. Chih B, et al. A ciliopathy complex at the transition zone protects the cilia as a privileged membrane domain. Nat Cell Biol 14, 61-72 (2012).

45. Wiegering $\mathrm{A}$, et al. Cell type-specific regulation of ciliary transition zone assembly in vertebrates. EMBO J 37, (2018).

46. Lambacher NJ, et al. TMEM107 recruits ciliopathy proteins to subdomains of the ciliary transition zone and causes Joubert syndrome. Nat Cell Biol 18, 122-131 (2016).

47. Yang TT, et al. Superresolution Pattern Recognition Reveals the Architectural Map of the Ciliary Transition Zone. Sci Rep 5, 14096 (2015).

48. Shiba D, Yokoyama T. The ciliary transitional zone and nephrocystins. Differentiation 83, S91-96 (2012).

49. Jensen VL, et al. Formation of the transition zone by Mks5/Rpgrip1L establishes a ciliary zone of exclusion (CIZE) that compartmentalises ciliary signalling proteins and controls PIP2 ciliary abundance. EMBO J 34, 2537-2556 (2015).

50. Wang L, et al. Rapid and high efficiency transformation of Chlamydomonas reinhardtii by square-wave electroporation. Biosci Rep 39, (2019).

51. Gonzalez-Ballester D, de Montaigu A, Galvan A, Fernandez E. Restriction enzyme site-directed amplification PCR: a tool to identify regions flanking a marker DNA. Anal Biochem 340, 330-335 (2005).

52. Wang $\mathrm{L}$, et al. Flagellar regeneration requires cytoplasmic microtubule depolymerization and kinesin-13. J Cell Sci 126, 1531-1540 (2013).

53. Meng D, Pan J. A NIMA-related kinase, CNK4, regulates ciliary stability and length. Mol Biol Cell 27, 838-847 (2016).

54. Lefebvre PA, Nordstrom SA, Moulder JE, Rosenbaum JL. Flagellar elongation and shortening in Chlamydomonas. IV. Effects of flagellar detachment, regeneration, and resorption on the induction of flagellar protein synthesis. J Cell Biol 78, 8-27 (1978).

55. Meng D, Cao M, Oda T, Pan J. The conserved ciliary protein Bug22 controls planar beating of Chlamydomonas flagella. J Cell Sci 127, 281-287 (2014). 
575 Fig. 1: Characterization of the C. reinhardtii tctn1 mutant.

a

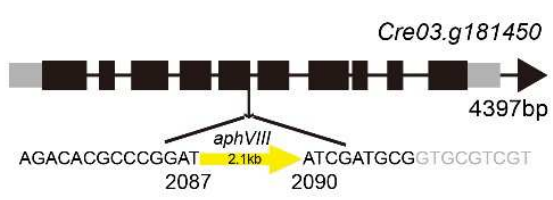

b

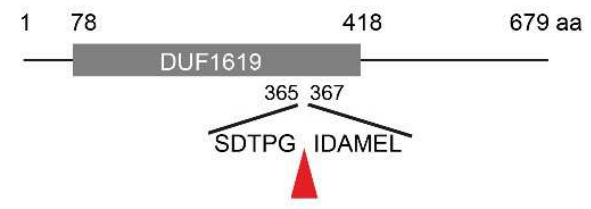

g

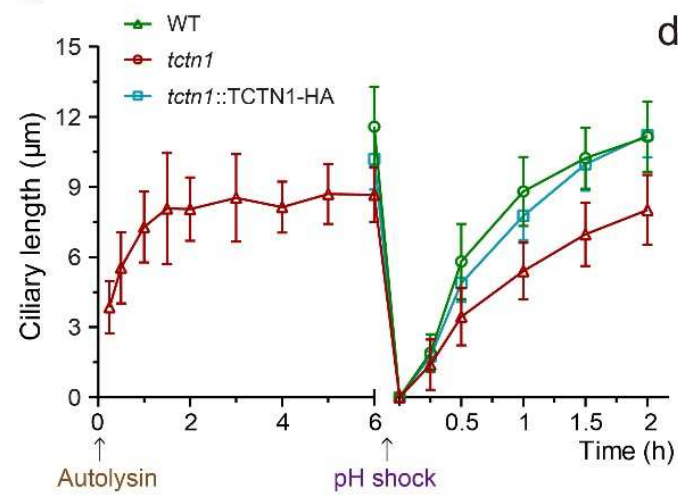

C

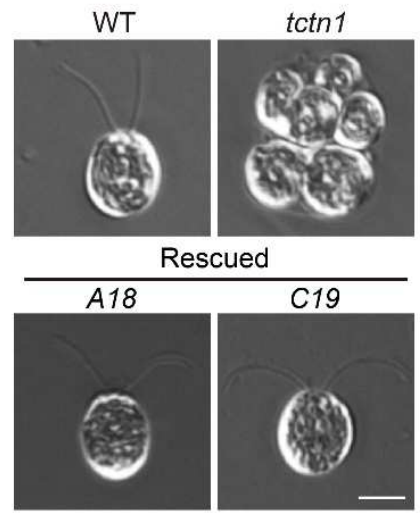

d

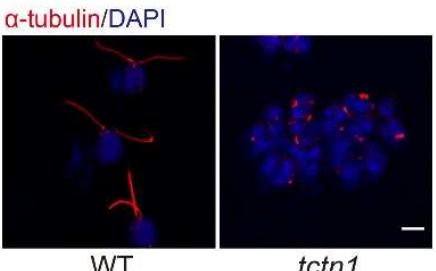

e

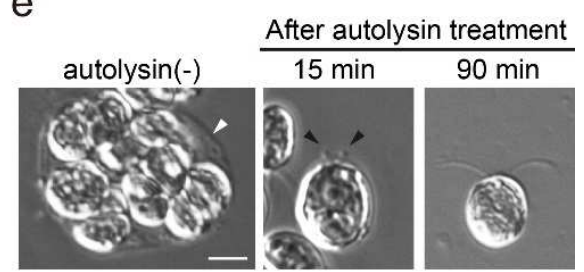

f

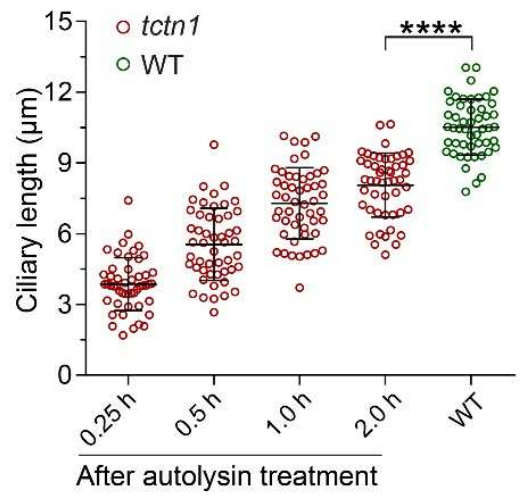

h

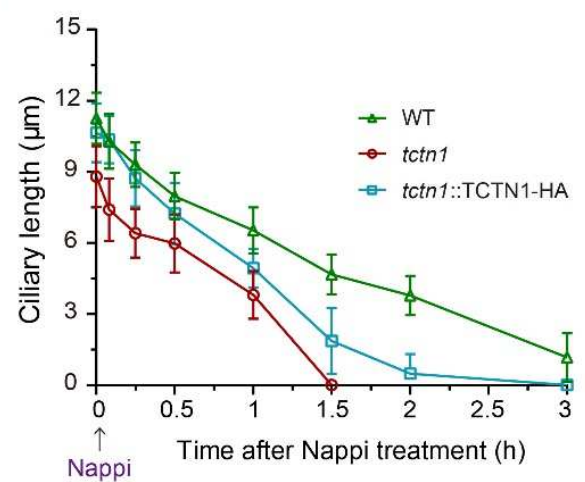

\section{i}

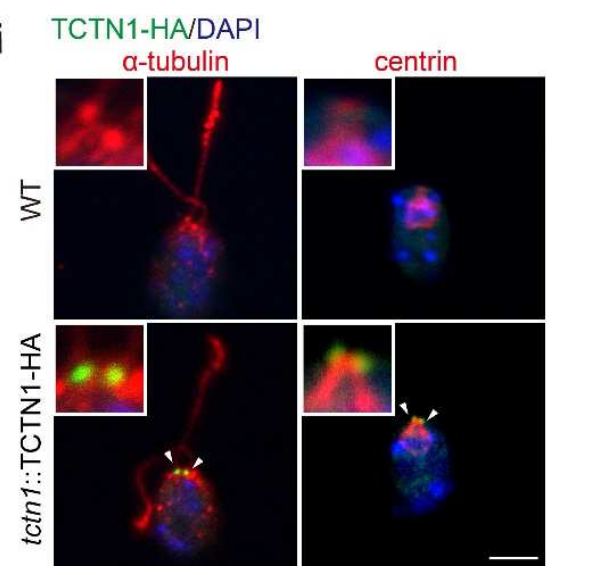

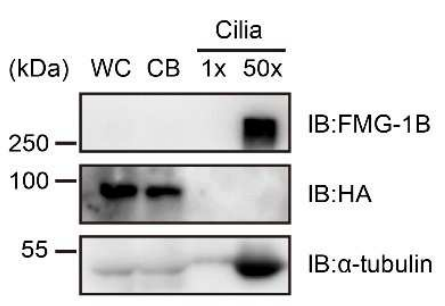

a Diagram of the gene structure of TCTN1 (Cre03.g181450) with the resistance DNA insertional site. The aphVIII DNA fragment $(\sim 2.1 \mathrm{~kb})$ was inserted in the fifth exon of TCTN1. The yellow box with the arrow indicates the insertional site. $\mathbf{b}$ Diagram of the domain structure of the TCTN1 protein. The TCTN1 protein (679 aa) contains the DUF1619 domain (78-418 aa) with unknown function. The red arrowhead marks the insertional site. c DIC images showing the ciliary phenotypes of WT, mutant, and rescued cells $(A 18, C 19)$. The tctn1 mutant are palmelloid (cells failed to hatch from the mother cell wall after mitosis). TCTN1 tagged with $3 \times \mathrm{HA}$ at the $\mathrm{C}$-terminus was transformed into tctn 1 to rescue the mutant. Scale bar, $5 \mu \mathrm{m}$. $\mathbf{d}$ Immunostaining images depicting the very short cilium within the palmelloid of tctn1. The red signals (by anti- $\alpha$-tubulin antibody) mark the cilia and the blue signals (by DAPI) mark the nucleus. Scale bar, $5 \mu \mathrm{m}$. e DIC images depicting the ciliary phenotype of $t c t n 1$ after treatment with autolysin. The tctn 1 cells released from the mother cell walls (white arrowhead) and elongated cilia, and occasionally with the bugles (black arrowheads) at the short ciliary tip. Scale bar, $5 \mu \mathrm{m}$. $\mathrm{f}$ Scatter plot showing the elongation of cilia of 
589 cells hatched from the palmelloid after autolysin treatment. Statistical significance was determined with an unpaired $590 t$ test. ${ }^{* * * *}, \mathrm{P}<0.0001$. $\mathrm{g}$ tctn 1 exhibited shorter cilia after hatching with autolysin and slower kinetics of ciliary 591 assembly. The arrow indicates the time point of autolysin treatment to release cells or $\mathrm{pH}$ shock treatment for 592 deciliation. $\mathbf{h}$ tctn1 exhibited faster kinetics of ciliary disassembly. The arrow indicates the time point of Nappi 593 treatment to induce ciliary shortening. i Immunostaining showing the localization of TCTN1 in the TZ. WT and the 594 rescued cells expressing TCTN1-HA were immunostained with HA (green) and a-tubulin (red, left) or centrin (red, 595 right) antibodies. The nucleus was stained with DAPI (blue). The arrowheads indicate the TZ at the ciliary base. 596 The insets show higher magnification views of the TZ region. Scale bar, $5 \mu \mathrm{m}$. $\mathbf{j}$ Immunoblot analysis of the 597 localization of TCTN1-HA in the rescued cell line. Cell body and cilia were isolated from the rescued cells . $1 \times$ (cilia) 598 represents an equal proportion of cilia to that of the cell body (two cilia per cell body). $50 \times$ (cilia) represents equal 599 cilia and cell body proteins. WC, whole cell. CB, cell body. Ciliary lengths are shown as the mean \pm SD of 50 cilia 600 and repeated three times in this figure.

601 
Fig. 2: Loss of TCTN1 causes TZ defects similar to those caused by loss of NPHP4 and CEP290.

a

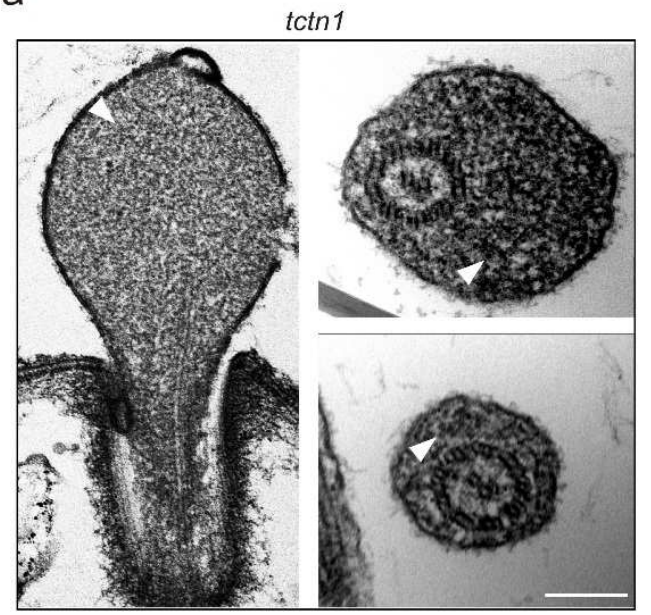

b

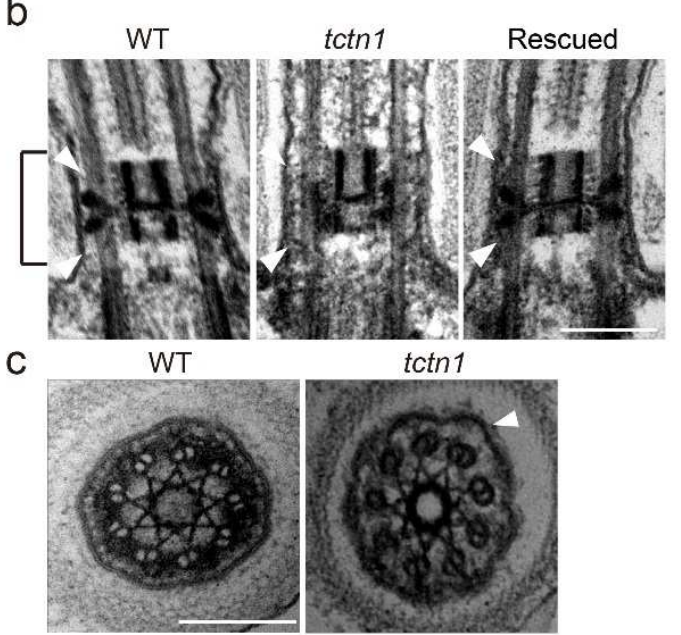

d

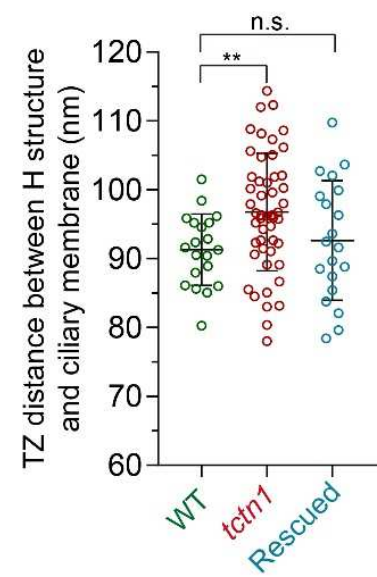

e

ac-tubulin/TCTN1-HA/

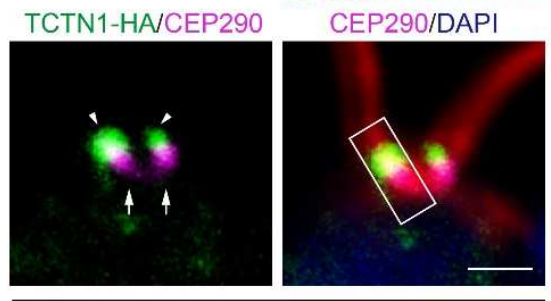

$\operatorname{tctn} 1:: \mathrm{TCTN} 1-\mathrm{HA}$

f

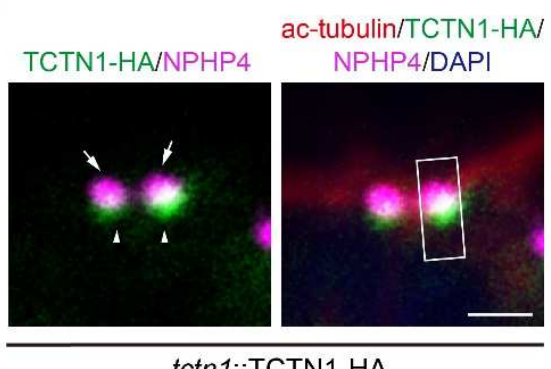

g
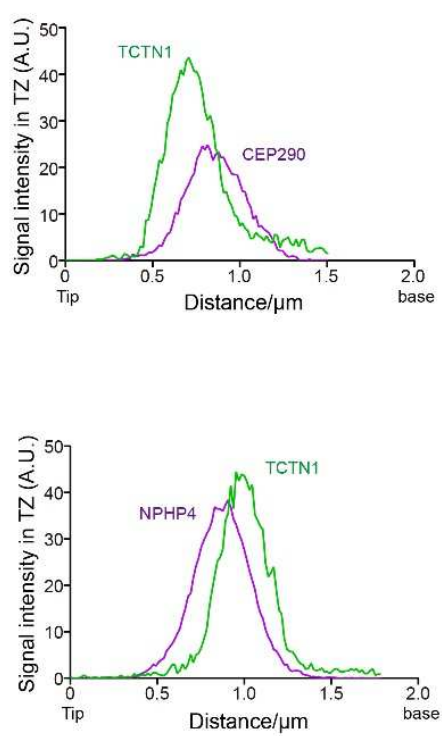

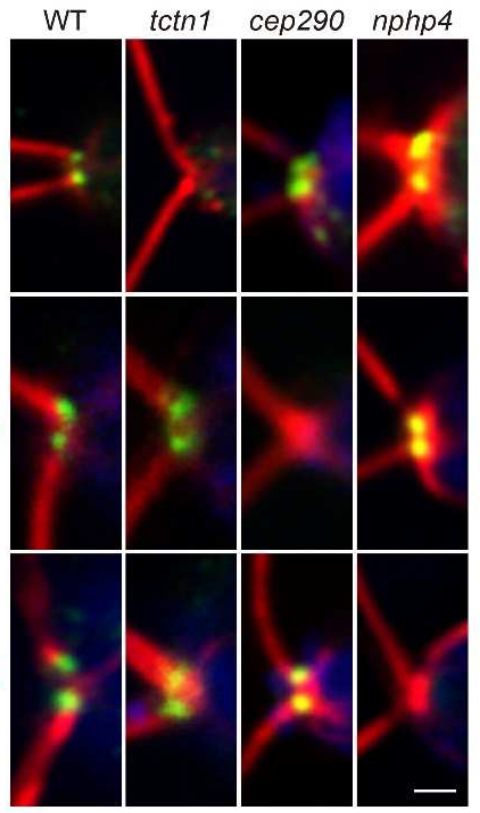

a EM images showing ciliary bulges with electron-dense material (white arrowheads) in a portion of tctn1 cells. Scale bar, $200 \mathrm{~nm}$. b, c Longitudinal sections and cross sections through the TZ (brackets) of WT, tctn1 and rescued cells. Y-links (white arrowheads) between the TZ microtubules and ciliary membrane were present in WT and rescued cells but were missing in tctn1 cells. Scale bar, $200 \mathrm{~nm}$. d Scatter plot depicting the distances between the $\mathrm{H}$ structure and the ciliary membrane in WT, tctn1, and rescued cells. Data are the mean $\pm \mathrm{SD}(\mathrm{n}=20)$. Statistical significance was determined with an unpaired $t$ test. n.s., not significant. ${ }^{* *}, \mathrm{P}<0.01$. e, $\mathbf{f}$ Immunostaining images and graphs depicting the colocalization of TCTN1 and NPHP4/CEP290. The rescued cells expressing TCTN1-HA were immunostained with anti-acetylated $\alpha$-tubulin (ac-tubulin, red), anti-HA (green) and anti-CEP290 (e, magenta) or anti-NPHP4 (f, magenta) antibodies. The nucleus was stained with DAPI (blue). The arrowheads and arrows indicate the localization of TCTN1-HA and CEP290/NPHP4, respectively. The scan plots of the rectangular gray value in the merged image show the relative intensities (A.U.) of the indicated proteins in the TZ. Scale bar, $1 \mu \mathrm{m}$. g Immunostaining depicting the TZ localization of TCTN1, NPHP4, and CEP290 in WT, tctn1, cep290, and nphp4 cells. Cells as indicated were immunostained with anti-acetylated $\alpha$-tubulin (ac-tubulin, red), anti-TCTN1/ CEP290/NPHP4 (green) antibodies. The nucleus was stained with DAPI (blue). Scale bar, $1 \mu \mathrm{m}$. 
Fig. 3: The integrity of TCTN1 is essential for its ciliary function.

a

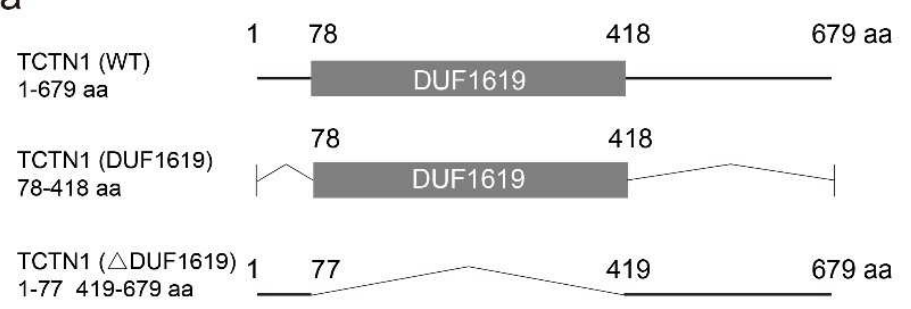

C

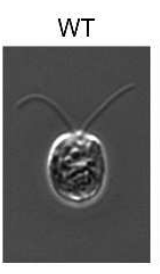

tctn 1

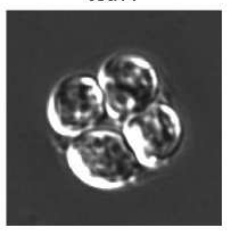

tctn1::TCTN1

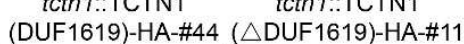

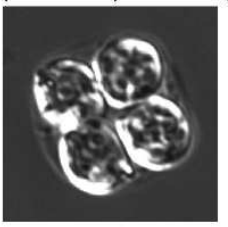

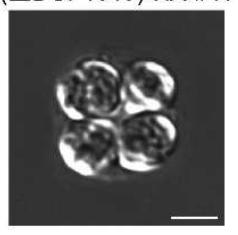

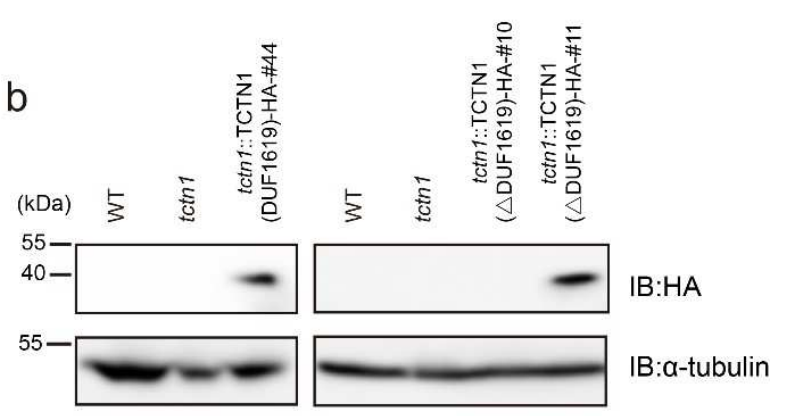

$d$

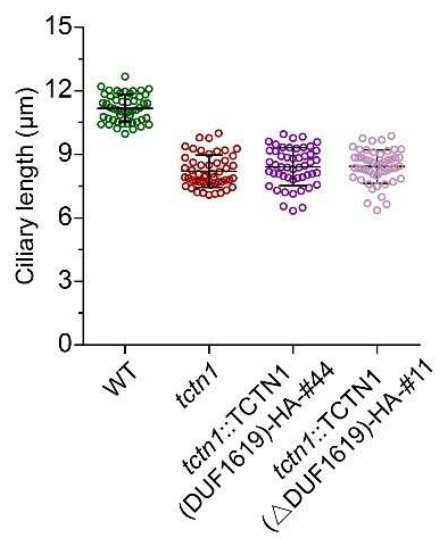

a Diagram of the domain structure and truncated mutants of TCTN1. The TCTN1 (DUF1619) protein contains the region of 78-418 aa of TCTN1, while the TCTN1 ( $\triangle$ DUF1619) protein contains the region of 1-77 aa and 419-679 aa of TCTN1. b Immunoblot analysis of the expression of truncated versions of TCTN1, denoted TCTN1 (DUF1619)-HA and TCTN1 ( $\triangle$ DUF1619)-HA. Cell lysates from the indicated cells were subjected to immunoblotting and probed with the indicated antibodies. WT and tctn1 cells were used as negative controls. $\alpha$-tubulin was used as a loading control. The positions of standard proteins and their molecular masses in $\mathrm{kDa}$ are indicated. c DIC images showing the ciliary phenotypes of WT, tctn1, and tctn 1 mutant cells expressing truncated TCTN1. Both tctn1 and the truncated TCTN1 expressing cell lines showed palmelloid form, suggesting the truncated TCTN1 could not rescue the phenotype of $t c t n 1$ mutant. Scale bar, $5 \mu \mathrm{m}$. d Scatter plot depicting the mean $\pm S D(n=50)$ of ciliary length of WT, tctn1, and cells expressing the truncated versions of TCTN1. 
Fig. 4: Loss of TCTN1 attenuates the ciliary gating role of the TZ.
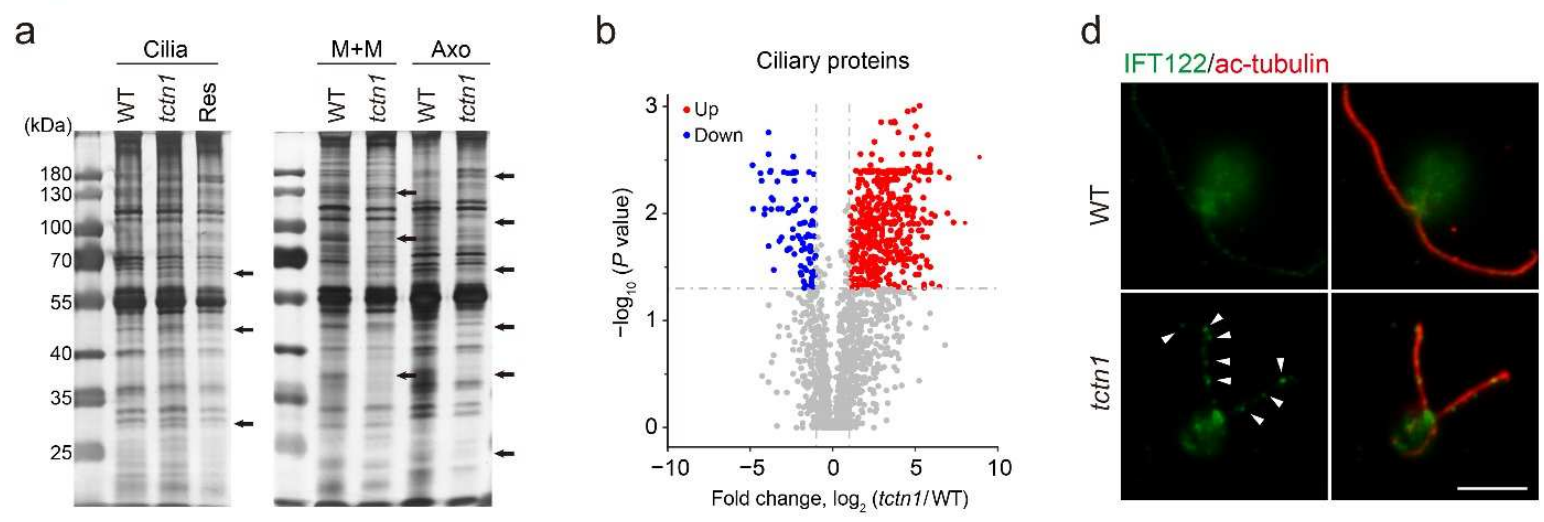

C

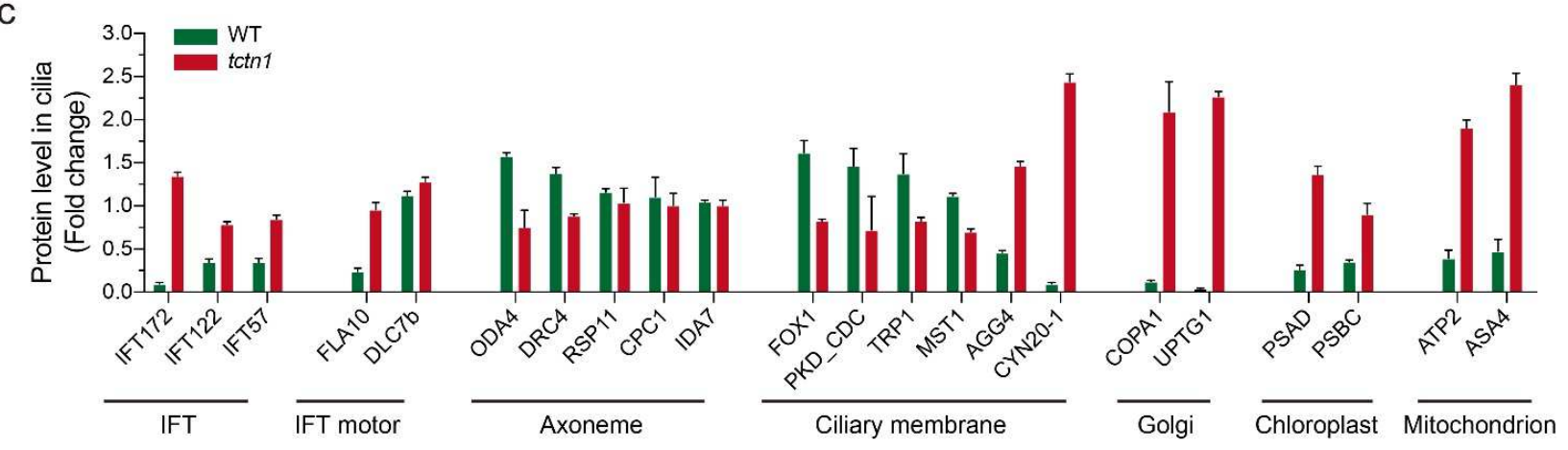

e

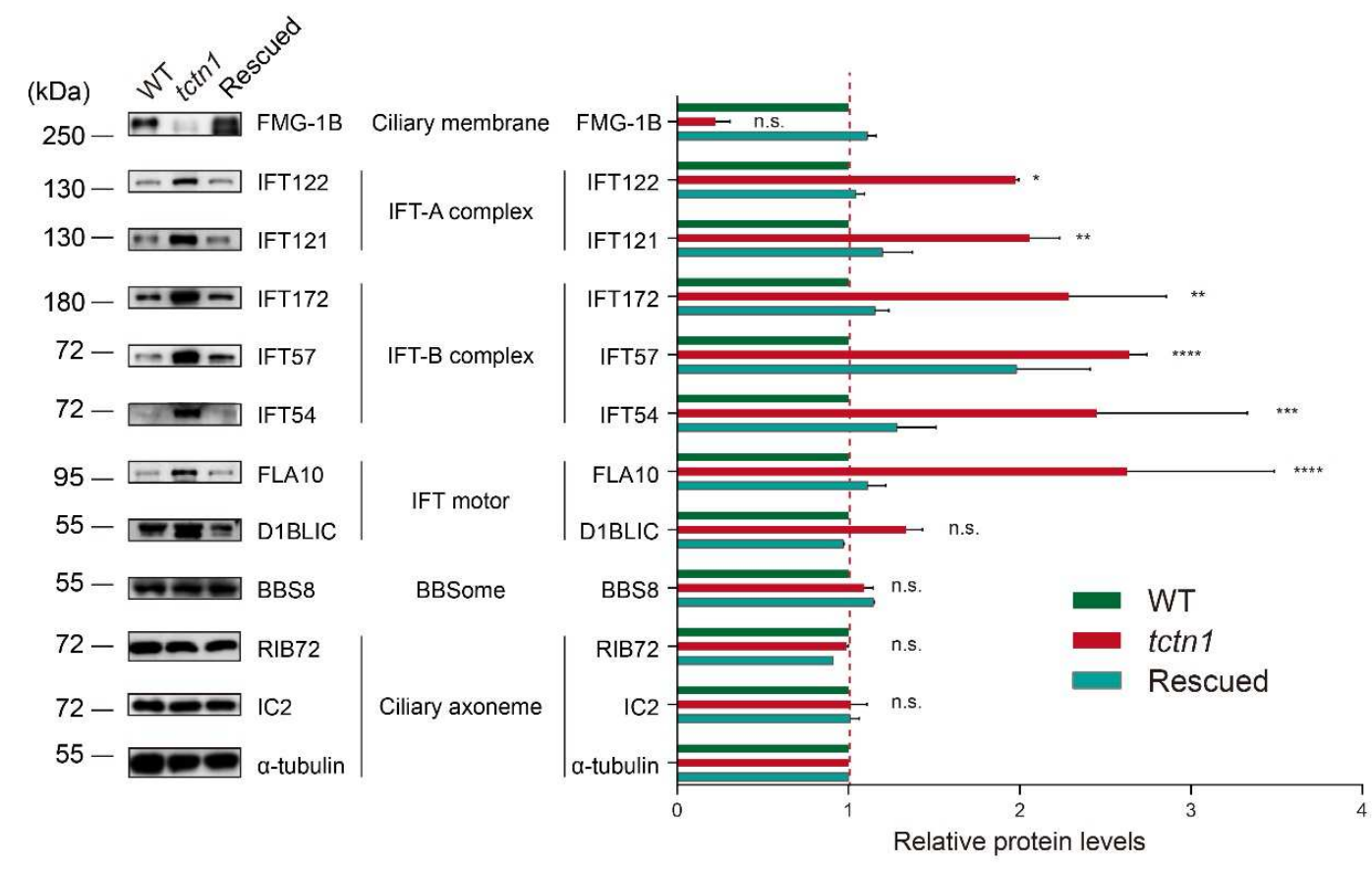

f
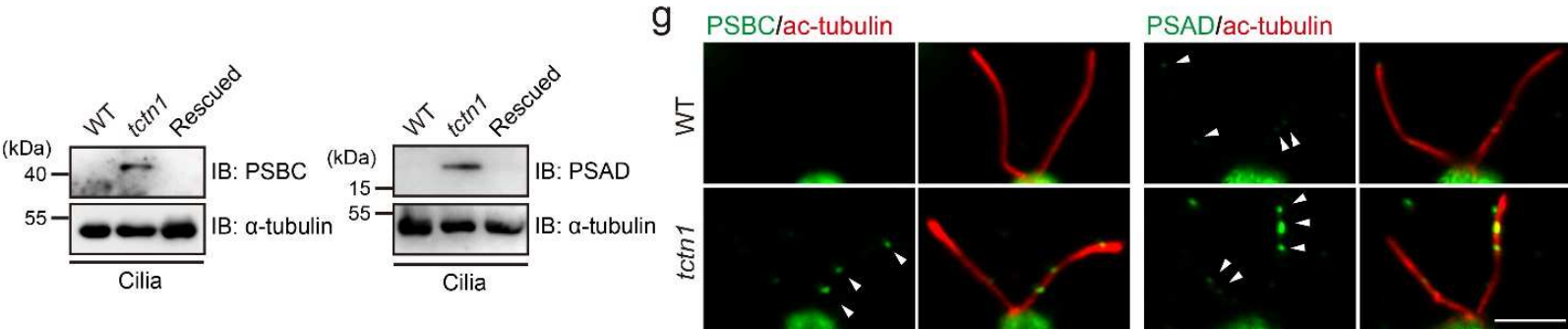
a SDS-PAGE and silver staining of isolated whole cilia or fractionations of cilia from WT, tctn1, and rescued cells expressing TCTN1-HA. The samples as indicated were separated on 10\% SDS-PAGE and then visualized by silver staining to observe the differences of the protein composition from WT, tctn1, and rescued cells. The positions of standard proteins and their molecular masses in $\mathrm{kDa}$ are indicated. Arrows indicate the differences among samples. Res, rescued cells. Cilia, ciliary samples. $M+M$, membrane and matrix samples of cilia. Axo, axonemal samples of cilia. b Volcano plot displaying the differentially expressed proteins in cilia between the WT and tctn1 mutant. The $X$ axis corresponds to the $\log _{2}$-fold change value of $t c t n 1 / \mathrm{WT}$, while the $Y$ axis corresponds to statistical significance in terms of the - $\log 10$ ( $P$ value). The red and blue dots represent the upregulated and downregulated proteins in cilia of the tctn1 mutant. c Column graph depicting the fold changes in the representative proteins in cilia between WT and tctn1. The representative proteins were selected from the mass spectrometry data in $\mathbf{b}$ and grouped into IFT, IFT motor, axoneme, ciliary membrane, Golgi, chloroplast, and mitochondrion proteins. d Immunostaining images displaying the enrichment of IFT122 particles in the cilium of tctn1. WT and tctn1 cells were immunostained with anti-IFT122 (green) and anti-acetylated a-tubulin (ac-tubulin, red) antibodies. The arrowheads indicate the accumulations of IFT122 in the cilium. Scale bar, $5 \mu \mathrm{m}$. e Immunoblot and statistical analysis of the isolated cilia from WT, tctn1, and rescued cells. The ciliary membrane protein (FMG-1B) was downregulated in cilia, while IFTA complex (IFT121 and IFT122), IFT-B complex (IFT54, IFT57, and IFT172), and IFT motor (FLA10 and D1BLIC) proteins were accumulated in tctn1 cilia, and the BBSome (BBS8) and ciliary axonemal protein (RIB72 and IC2) levels were comparable with those in WT. $\alpha$-tubulin was used as a loading control. The positions of standard proteins and their molecular masses in $\mathrm{kDa}$ are indicated. The graph showing the gray value of the immunoreactive bands was prepared using the mean \pm SEM $(n=2)$. Statistical significance to WT group was determined using two-way ANOVA. n.s., not significant. ${ }^{*}, \mathrm{P}<0.1$. ${ }^{* *}, \mathrm{P}<0.01 .{ }^{* * *}, \mathrm{P}<0.001 .{ }^{* * * *}, \mathrm{P}<0.0001$. $\mathrm{f} \mathrm{Immunoblot} \mathrm{of} \mathrm{cilia} \mathrm{isolated} \mathrm{from}$ WT, tctn1, and rescued cells. The antibodies against photosystem proteins (PSBC and PSAD) were used for the confirmation of proteomics results from $\mathbf{b}$. The positions of standard proteins and their molecular masses in $\mathrm{kDa}$ are indicated. $\mathbf{g}$ Immunostaining images showing enrichment of photosystem proteins (PSBC, PSAD) in tctn1. WT and $t c t n 1$ cells were immunostained with anti-PSBC (green, left), or anti-PSAD (green, right) and anti-acetylated $\alpha-$ tubulin (ac-tubulin, red) antibodies. The non-ciliary proteins were transported and accumulated within the cilium. The arrowheads indicate the accumulation of photosystem proteins in the cilium. Scale bar, $5 \mu \mathrm{m}$. 
Fig. 5: Proteins differentially regulated in cilia by different TZ proteins.

a
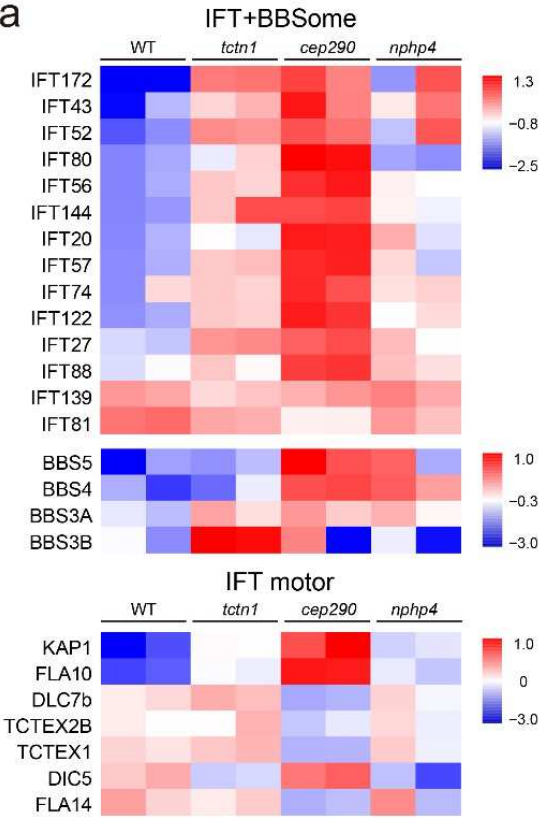

d

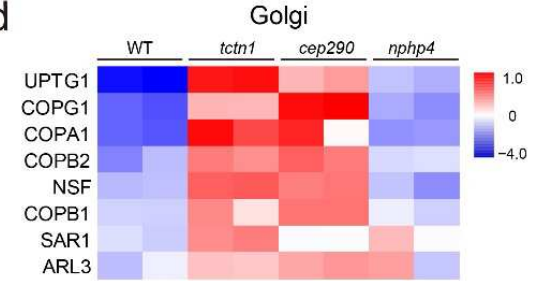

e

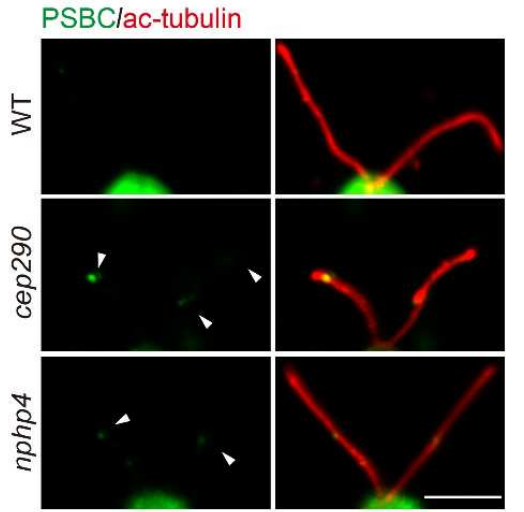

b
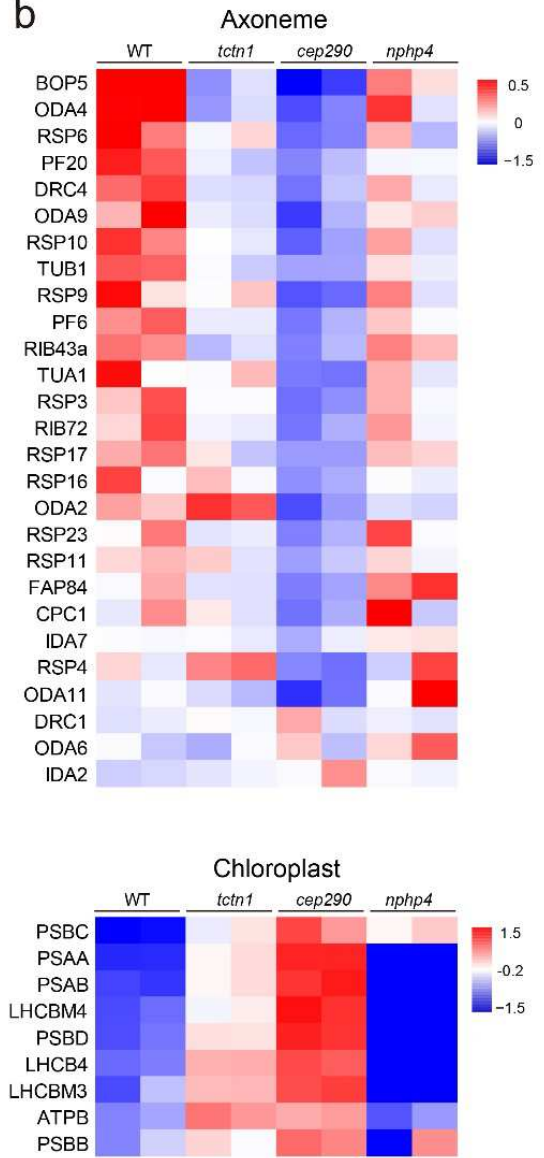

f
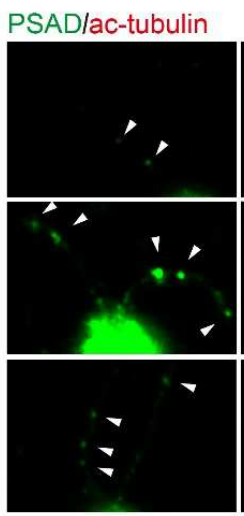

C
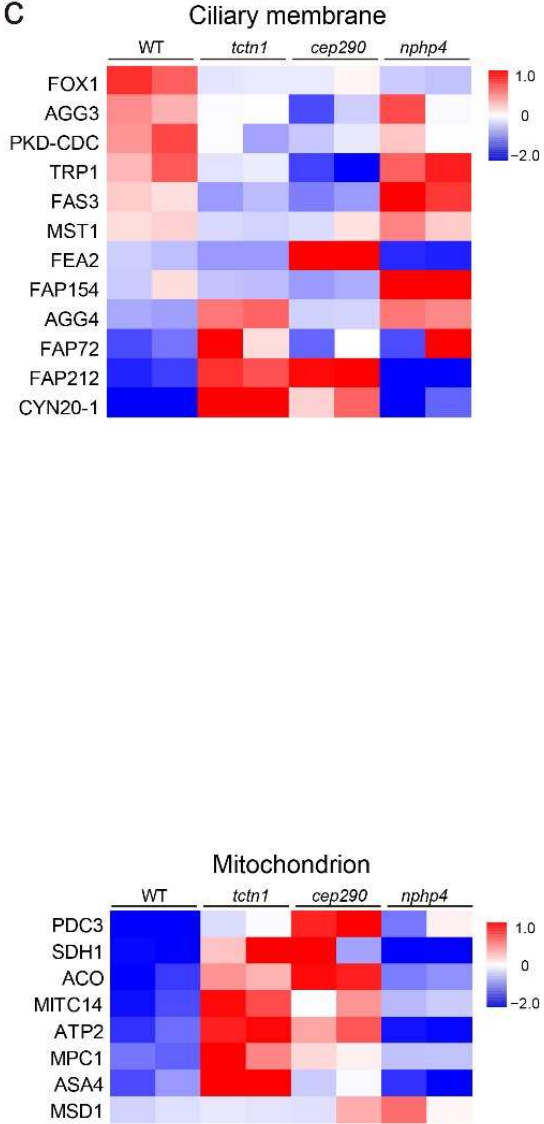

g

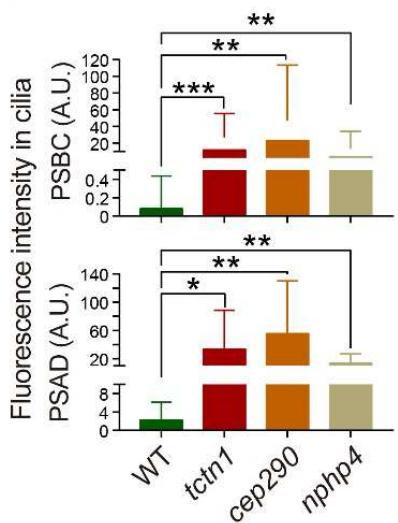

a-d Heatmap of representative groups of proteins identified from ciliary proteomics analysis of cilia from WT, tctn1, cep290, and nphp4 cells (two replicates for each strain, column). Representative proteins were selected from the mass spectrometry data in Fig. S6A and grouped into IFT and BBSome (a, top), IFT motor (a, bottom), Axoneme (b), Ciliary membrane (c), Golgi (d, left), Chloroplast (d, middle) and Mitochondrion (d, right) proteins. e, $\mathbf{f}$ Immunostaining images displaying the enrichment of photosystem proteins (PSBC and PSAD) in TZ mutants (cep290 and nphp4). WT and TZ mutants were immunostained with anti-PSBC (e, green), or anti-PSAD (f, green) and anti-acetylated a-tubulin (ac-tubulin, red) antibodies. The non-ciliary proteins were transported and accumulated in cilia of TZ mutant cells. The arrowheads indicate the accumulations of photosystem proteins in the 
674 cilium. Scale bar, $5 \mu \mathrm{m}$. g Column graphs show the ciliary fluorescence intensity of PSBC or PSAD. Data are the 675 mean $\pm \operatorname{SD}(n=20)$. Statistical significance was determined with an unpaired $t$ test. ${ }^{*}, P<0.1 .{ }^{* *}, P<0.01 .{ }^{* * *}, P<0.001$. 
Fig. 6: The formation of ciliary ectosomes is regulated by the integrity of the TZ during gamete mating 680 events.

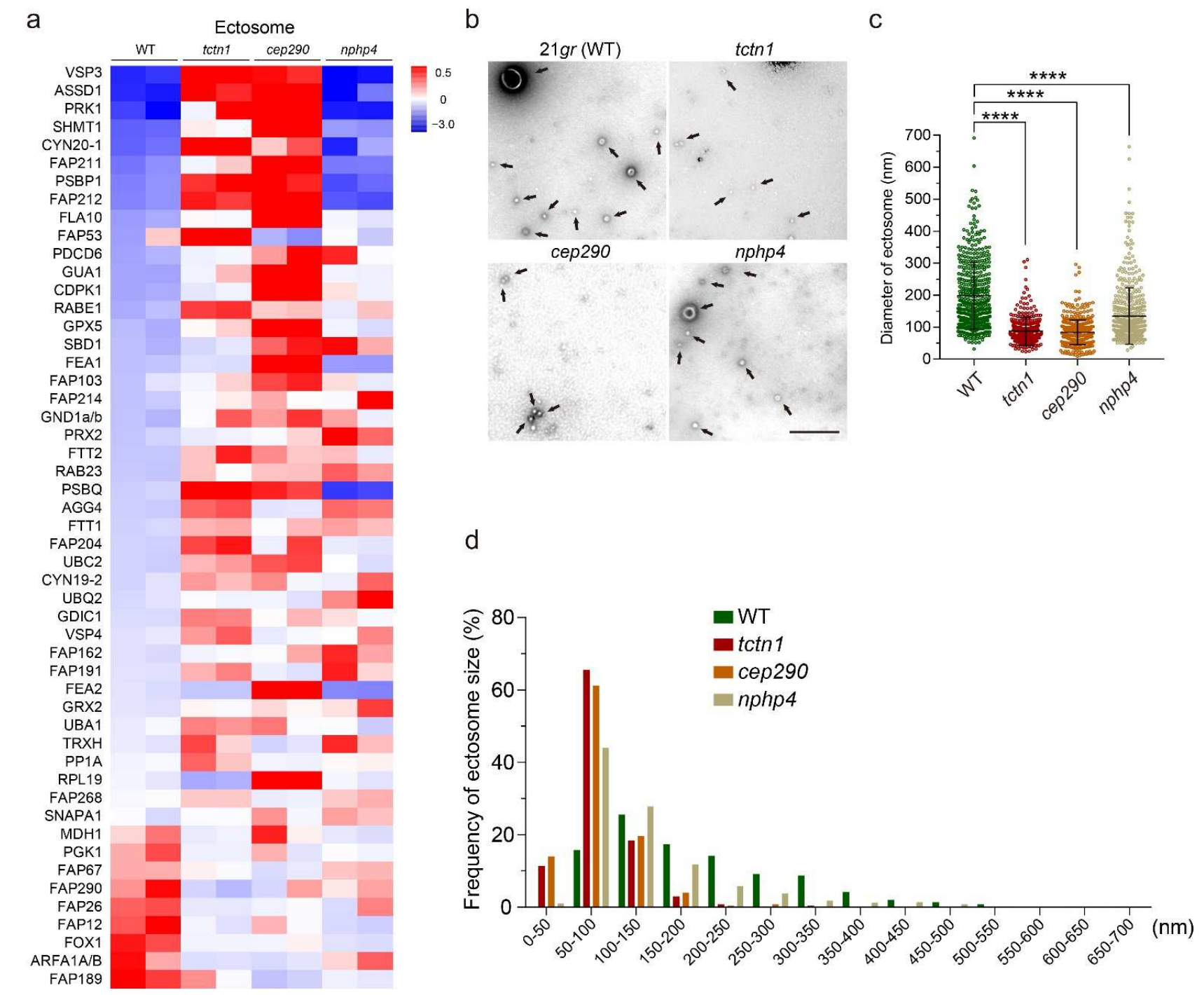

a Heatmap of representative ectosome related proteins identified from ciliary proteomics analysis of cilia from WT, tctn1, cep290, and $n p h p 4$ cells (two replicates for each strain, column). Representative proteins were selected from the mass spectrometry data in Fig. S6A. b Negative stain transmission electron micrographs of purified ciliary ectosomes (black arrows) from gametes in the mating process. 21 $\mathrm{gr}$ (WT), ectosomes from mating supernatant of $21 \mathrm{gr} \times 6145 \mathrm{c}$; tctn1, ectosomes from mating supernatant of tctn1 $\times 6145 \mathrm{c}$; cep290, ectosomes from mating supernatant of cep290 × 6145c; nphp4, ectosomes from mating supernatant of $n p h p 4 \times 6145 \mathrm{c}$. Scale bar, $500 \mathrm{~nm}$. c Scatter plot showing the diameters of ectosomes shedded from the cilia of different mating gametes. The sizes of the ectosomes $(n=500)$ described in $\mathbf{b}$. $\mathbf{d}$ Graph showing the distribution of ciliary ectosomes of different sizes purified from $21 \mathrm{gr} \times 6145 \mathrm{c}$, tctn $1 \times 6145 \mathrm{c}$, cep290 $\times 6145 \mathrm{c}$, and $n p h p 4 \times 6145$ described in $\mathbf{b}$. 
a

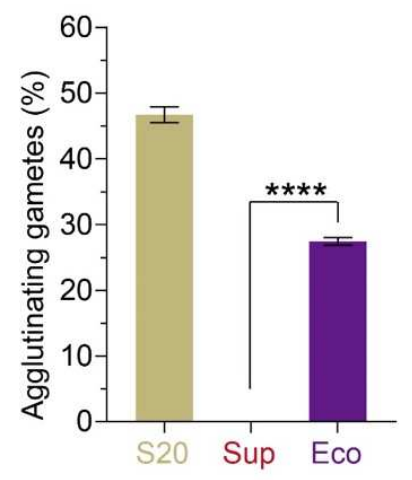

C

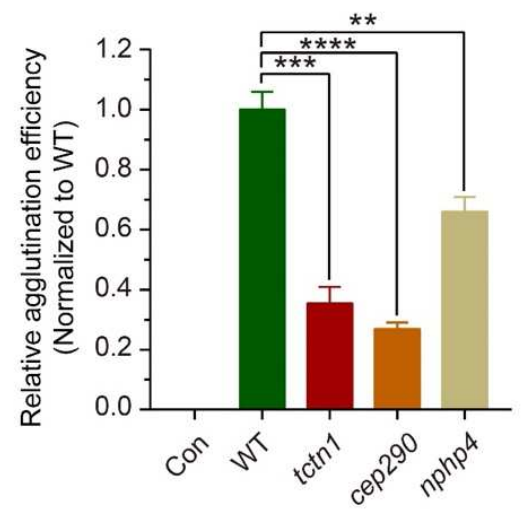

b

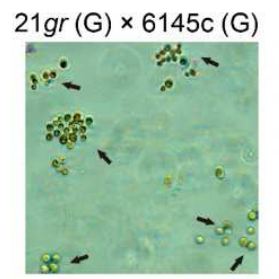

$\mathrm{Eco}+6145 \mathrm{c}(\mathrm{V})$
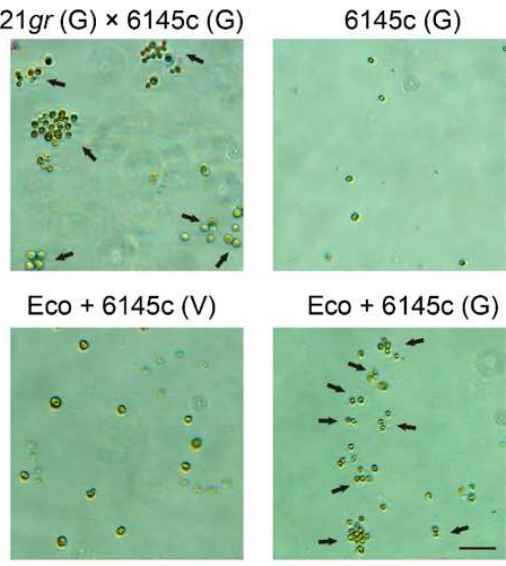

$E c o+6145 c(G)$

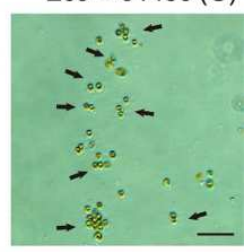

d

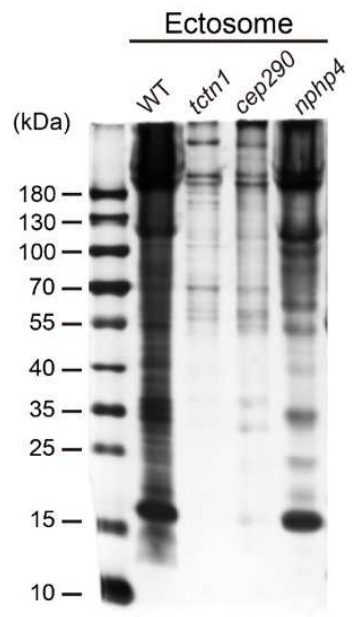

a Column graph depicting the agglutinating gametes number (\%) from $6145 \mathrm{c}(\mathrm{mt}-)$ mixed with the supernatant (S20), the final supernatant (Sup) and ectosome pellet (Eco) from gamete mating culture $(21 \mathrm{gr} \times 6145 \mathrm{c})$. Data are shown as the mean $\pm \mathrm{SD}(\mathrm{n}=3)$. Statistical significance was determined with an unpaired $t$ test. ${ }^{* * *}, \mathrm{P}<0.0001$. b $\mathrm{PH}$ images showing the agglutination of gametic $6145 \mathrm{c}(\mathrm{mt}-)$ induced by mixing with the ectosomes (Eco). Gametic $6145 \mathrm{c}$ alone, vegetative $6145 \mathrm{c}$ with Eco, and $21 \mathrm{gr} \times 6145 \mathrm{c}$ were showed as the negative and positive controls respectively. The black arrows indicate the agglutination of gametic cells. G, gametic cells. V, vegetative cells. Scale bar, $20 \mu \mathrm{m}$. c Column graph displaying the agglutination efficiency (normalized to WT) of gametic 6145c (mt-) mixed with the ectosome pellet (Eco) from gamete mating cultures $(21 \mathrm{gr} \times 6145 \mathrm{c}$, tctn $1 \times 6145 \mathrm{c}$, cep $290 \times 6145 \mathrm{c}, n p h p 4$ $\times 6145 \mathrm{c}$ ). The group of vegetative $6145 \mathrm{c}$ with Eco was as the negative control (Con). $\mathbf{d}$ Silver-stained SDS-PAGE gel showing protein variations in ectosomes isolated from the plus mating type gamete $(21 \mathrm{gr}, \mathrm{tctn} 1, \mathrm{cep} 290, \mathrm{nphp} 4)$ with minus mating type gamete (6145c). The indicated ciliary ectosomes purified from the same amount of cells were separated on $4 \% \sim 20 \%$ SDS-PAGE and then stained with silver staining to visualize the different amounts and compositions of proteins in different ectosomes. The positions of standard proteins and their molecular masses in $\mathrm{kDa}$ are indicated. Data are presented as the mean $\pm \mathrm{SD}$ in this figure. Statistical significance was determined with an unpaired $t$ test. ${ }^{* *}, \mathrm{P}<0.01 .{ }^{* * *}, \mathrm{P}<0.001 .{ }^{* * * *}, \mathrm{P}<0.0001$. 
a

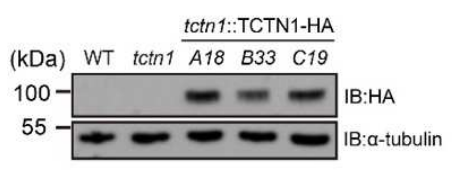

C

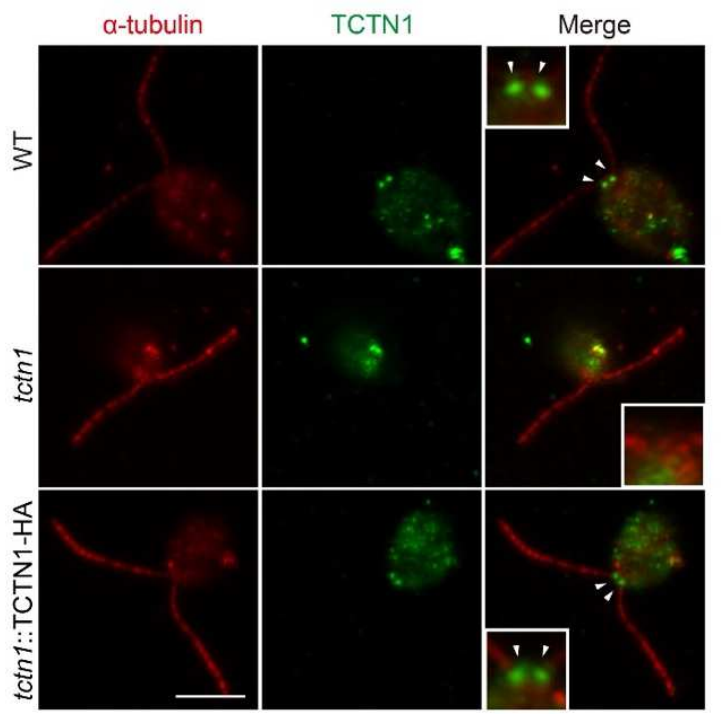

b

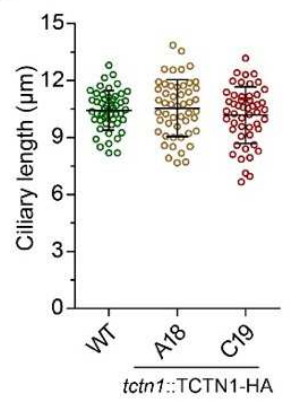

d

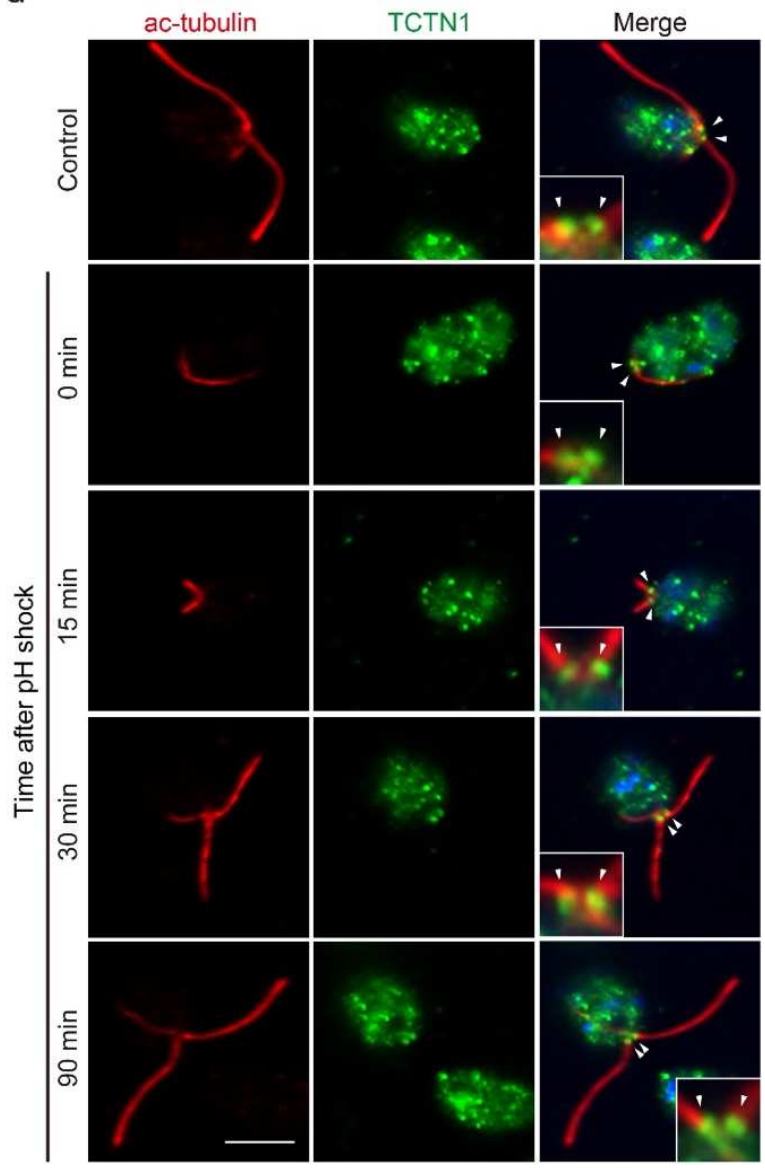

a Immunoblot analysis of the expression of TCTN1-HA in the WT, tctn1, and rescued cells. Cell lysates from the indicated cells were subjected to immunoblotting probed with the indicated antibodies. WT and tctn1 cells were used as negative controls. a-tubulin was used as a loading control. The positions of standard proteins and their molecular masses in $\mathrm{kDa}$ are indicated. $\mathbf{b}$ Scatter plot showing the mean $\pm \mathrm{SD}$ of ciliary length $(\mathrm{n}=50)$ in the WT, tctn1, and rescued cells (A18 and C19). c Immunostaining images depicting the immunospecificity of the antiTCTN1 antibody and localization of TCTN1 in the TZ. WT, tctn1, and the rescued cells expressing TCTN1-HA were immunostained with anti-TCTN1 (green) and anti- $\alpha$-tubulin (red) antibodies. The arrowheads indicate the TZ at the ciliary base. The insets show the higher magnification views of the TZ region in the merge image. Scale bar, $5 \mu \mathrm{m}$. d Immunostaining images illustrating that the localization of TCTN1 in the TZ was not changed during ciliary regeneration. Cells were subjected to $\mathrm{pH}$ shock to induce deciliation and collected for immunostaining with antiTCTN1 (green) and anti-acetylated a-tubulin (ac-tubulin, red) antibodies at the indicated time point. The nucleus was stained with DAPI (blue). The arrowheads indicate the TZ at the ciliary base. The insets show higher magnification views of the TZ region in the merged image. Scale bar, $5 \mu \mathrm{m}$. 
a
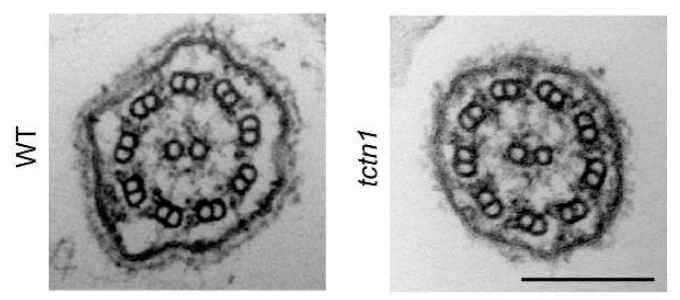

d

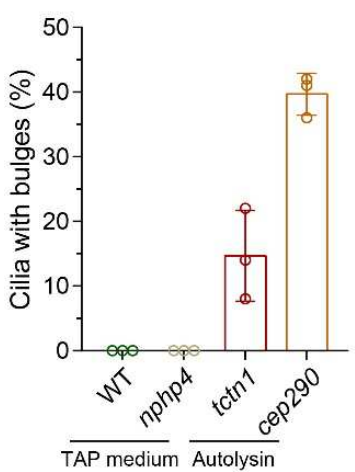

b

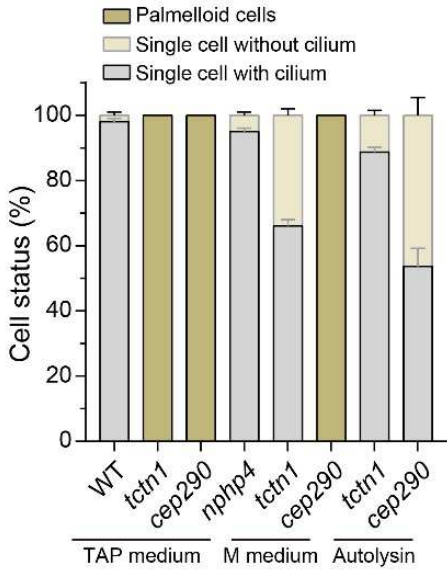

C

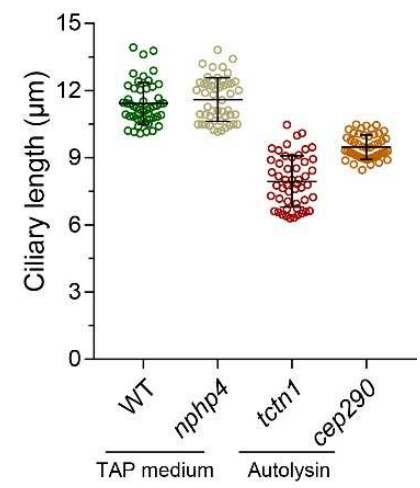

e

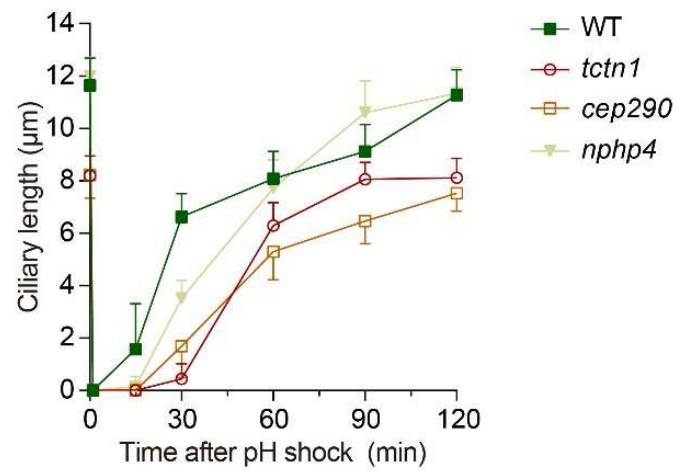

a TEM images showing cross sections through the cilia of WT and tctn1 cells. Scale bar, $200 \mathrm{~nm}$. b Column graph showing the cell status (\%) under different conditions (TAP medium, M medium, or after autolysin treatment) for WT, tctn1, cep290, and nphp4 cells. c Scatter plot showing the average ciliary length under different conditions (TAP medium or after autolysin treatment) for WT, tctn1, cep290, and nphp4 cells. d Column graph summarizing the percentages of cilia with bulges (\%) in WT, tctn1, cep290, and nphp4 cells. The palmelloid cells (tctn1 and cep290) were treated with autolysin for hatching before bulge counting. e Ciliary regeneration after deciliation by $\mathrm{pH}$ shock of WT, tctn1, cep290, and nphp4 cells. The palmelloid cells (tctn1 and cep290) were treated with autolysin and grown for $3 \mathrm{~h}$ before the deciliation assay and ciliary length analysis. Data are shown as the mean \pm SD $(n=50$ for the ciliary length; $n=200$ for the cell numbers) in this figure. 
Supplementary Figure 3. TCTN1 integrity is required for ciliary assembly.

a

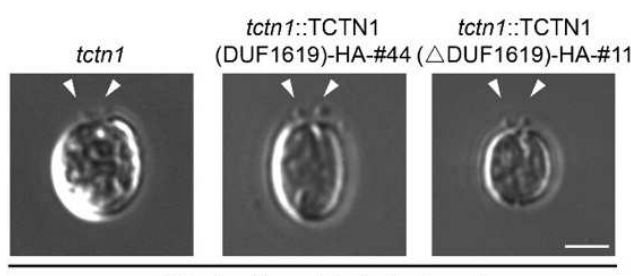

20 min after autolysin treatment b

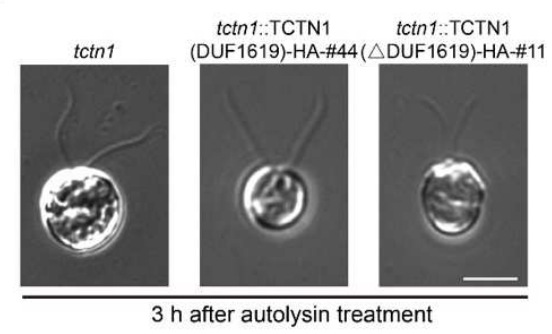

C

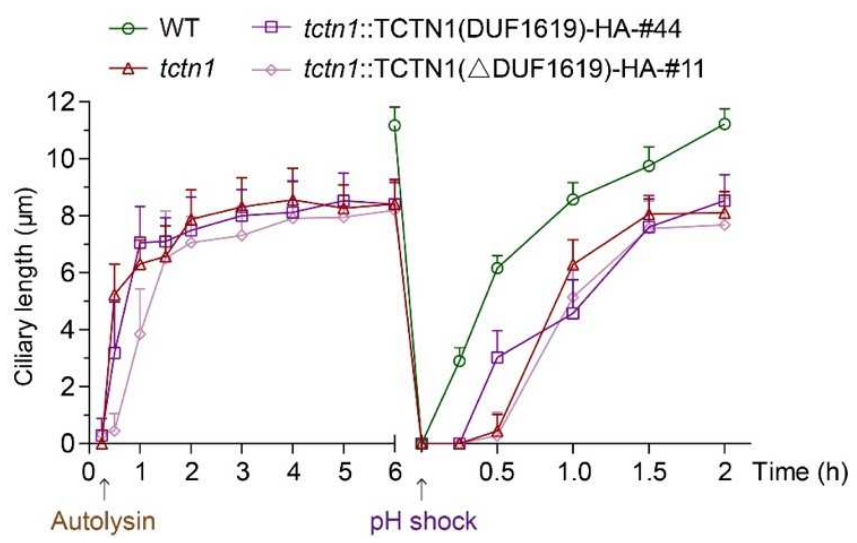

a DIC images showing the bulges at the tips of cilia in tctn1 and in cells expressing the truncated versions of TCTN1. Both tctn1 and cells expressing truncated TCTN1 hatched after autolysin treatment and showed bulges at the ciliary tips. Scale bar, $5 \mu \mathrm{m}$. b DIC images showing the elongated cilia in tctn1 and cells expressing truncated versions of TCTN1 hatched with autolysin. Scale bar, $5 \mu \mathrm{m}$. c The tctn1 cells and the cells expressing truncated versions of TCTN1 exhibited shorter cilia after treatment with autolysin and slower kinetics of ciliary assembly. The arrow indicates the time point of autolysin treatment to release cells or $\mathrm{pH}$ shock treatment for deciliation. Ciliary length is presented as the mean $\pm S D(n=50)$. 
a

IFT-A subunit

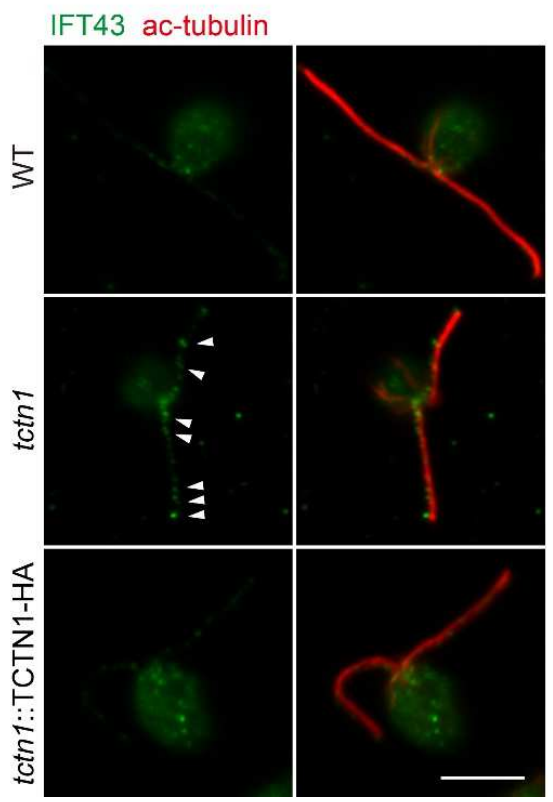

b

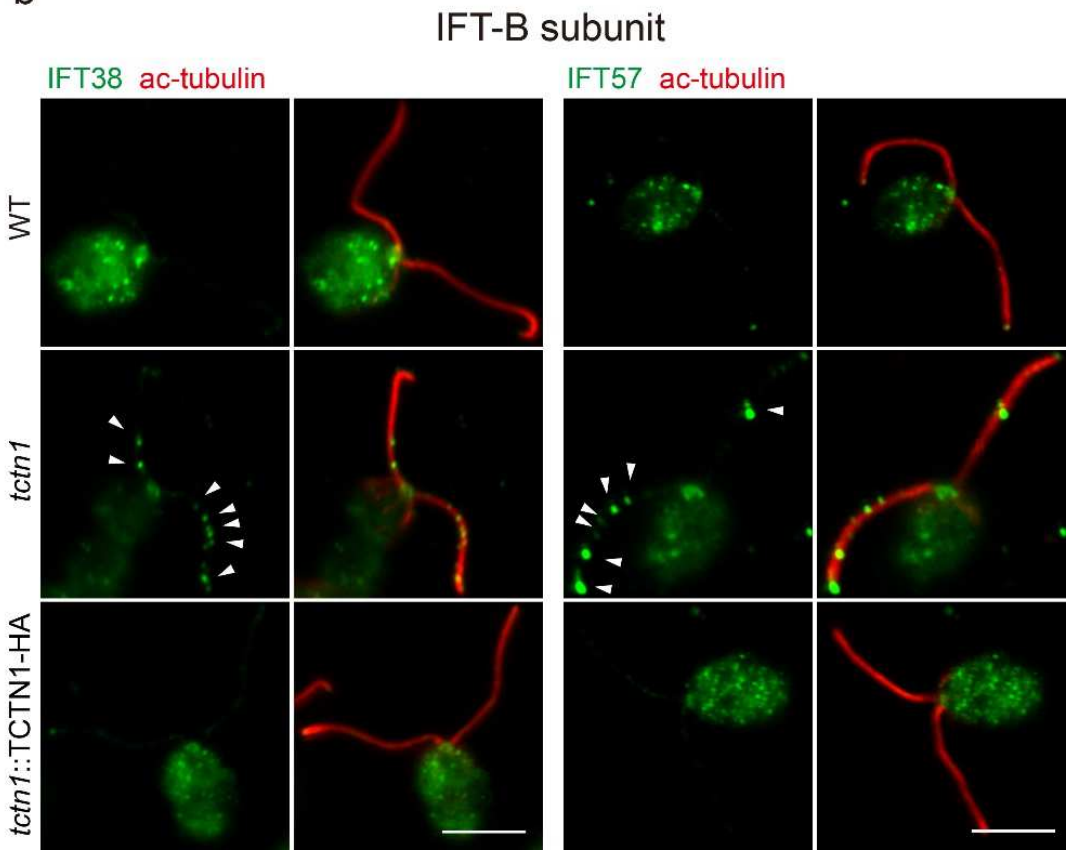

a, b Immunostaining images displaying the enrichment of IFT subunits in tctn1 cells. WT, tctn1, and rescued cells were immunostained with anti-IFT43 (a, green), or anti-IFT38 (b, left, green), or anti-IFT57 (b, right, green), and anti-acetylated $\alpha$-tubulin (ac-tubulin, red) antibodies. The arrowheads indicate the accumulation of IFT particles in 760 the cilium. Scale bar, $5 \mu \mathrm{m}$. 
762 Supplementary Figure 5. PSBC and PSAD staining reveal cup-shaped chloroplasts.

a

PSBC ac-tubulin

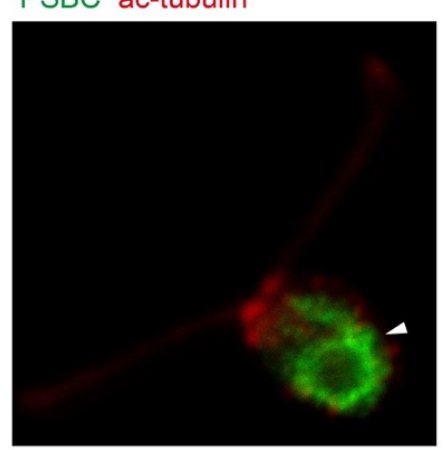

WT cells, Cup-shaped Chloroplasts

b

PSAD ac-tubulin

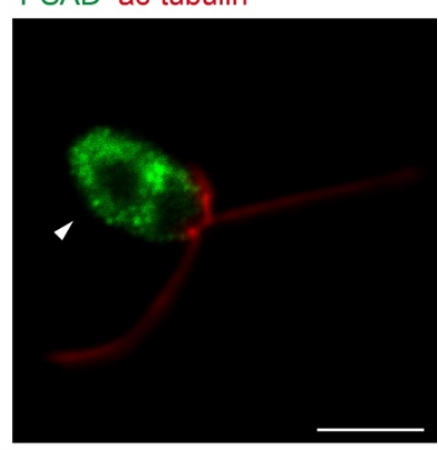

a, b Immunofluorescence images demonstrating the validity of antibodies against the photosystem proteins PSBC and PSAD in the chloroplast of WT cells. WT cells were immunostained with anti-PSBC (a, green), or anti-PSAD (b, green), and anti-acetylated a-tubulin (ac-tubulin, red) antibodies. The arrowheads show the cup-shaped chloroplasts. Scale bar, $5 \mu \mathrm{m}$. 
Supplementary Figure 6. The misregulated proteins in different TZ mutants (tctn1, cep290, and nphp4).

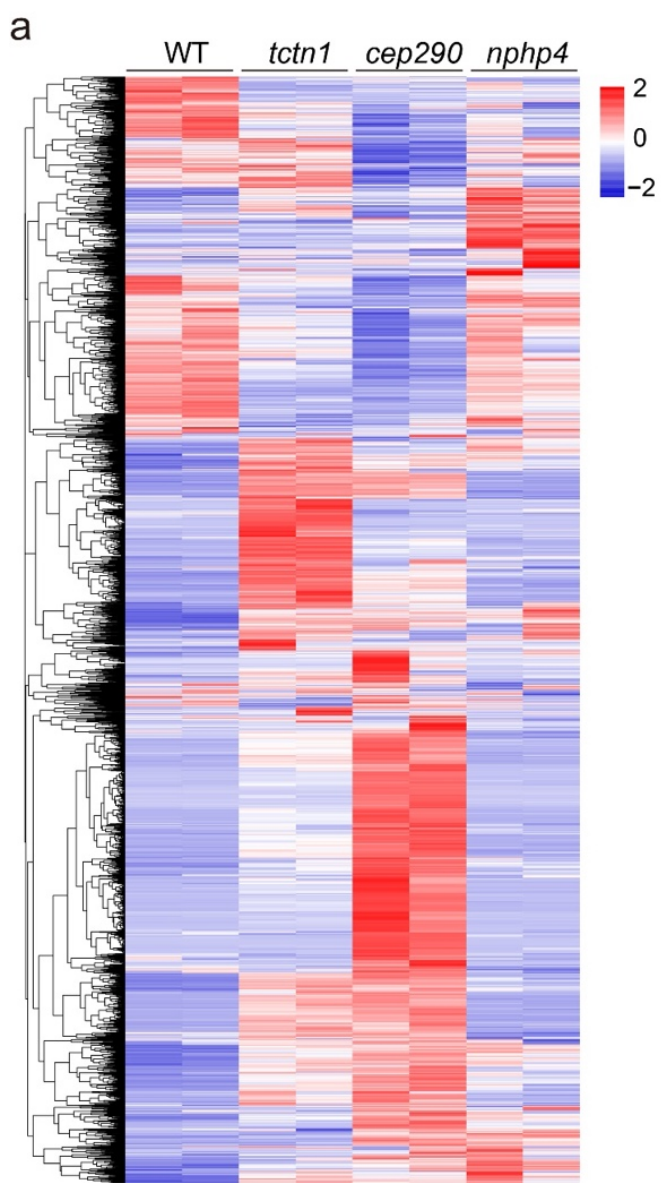

b

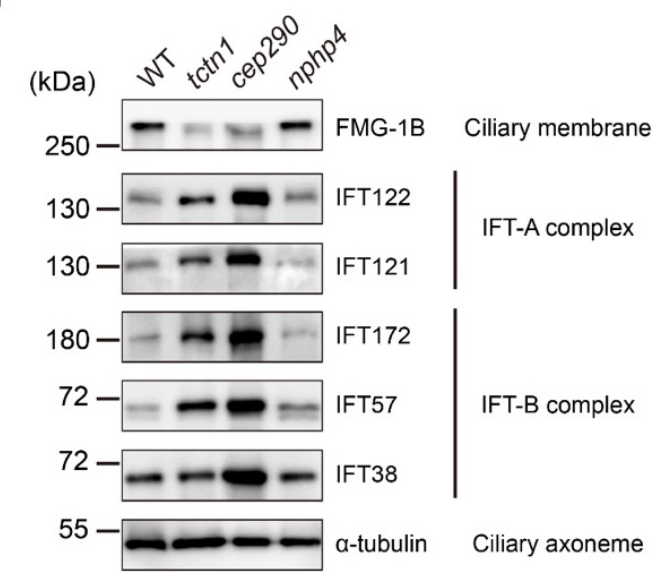

a Heatmap of all 2845 proteins identified in cilia from WT, tctn1, cep290, and nphp4. Fold changes in the expression of proteins from two biological replicates of these four strains are $\log _{2}$ transformed and displayed as colors ranging from red (upregulated) to blue (downregulated). b Immunoblot of cilia isolated from WT, tctn1, cep290, and nphp4 cells. Antibodies against FMG1-B, IFT-A complex (IFT121 and IFT122), and IFT-B complex (IFT38, IFT57, and IFT172) were used to confirm the proteomics results shown in A. $\alpha$-tubulin was used as a loading control. The positions of standard proteins and their molecular masses in $\mathrm{kDa}$ are indicated. 